\title{
EL IMPACTO DE LOS FACTORES CRÍTICOS DE ÉXITO EN LA IMPLEMENTACIÓN DE SISTEMAS INTEGRADOS DE ERP
}

\author{
Miguel Maldonado \\ Candidato doctoral en Administración y Dirección \\ de Empresas Esade-ESAN (España y Perú) \\ maldonado_pda606@esan.edu.pe
}

\section{Resumen}

El análisis del éxito de la implementación de los sistemas integrados de planificación de los recursos empresariales (ERP por sus siglas en inglés) es un tema que, aunque reciente, tiene mucha relevancia para la comunidad de tecnología de la información (TI). Sin embargo, y como resultado de esta novedad, la mayor parte de la investigación existente se circunscribe a metodologías cualitativas o estudios descriptivos orientados, sobre todo, a grandes empresas o corporaciones. En esta investigación se presenta un modelo que pretende revelar los factores determinantes del éxito de la implementación de ERP en la pequeña y la mediana empresa latinoamericana. La validación empírica de este modelo permitió verificar relaciones significativas entre adiestramiento y habilidades en TI en la empresa y el éxito de la implementación, medido por el tiempo requerido para esta. Se verificó también el impacto de este tiempo sobre la percepción de la satisfacción global de las empresas con la implementación del ERP. La investigación concluye con recomendaciones para su aplicación y sugiere también potenciales áreas de investigación adicionales inspiradas en un sorprendente resultado colateral obtenido.

Palabras clave: Sistemas ERP, sistemas integrados, enterprise resource planning, factores críticos de éxito, éxito de los ERP en las pyme.

\begin{abstract}
Although is a recent topic, the analysis of the success of the integrated systems implementation of the enterprise resource planning (ERP) is a topic that has a lot of relevance for the information technology (IT) community. However, as a result of this novelty, most of the existent investigation is limited to qualitative methodologies or descriptive studies oriented to big companies or corporations mainly. This investigation presents a model that seeks to reveal the determining factors of the success of ERP implementation in a small and medium Latin American company. The validation of this empiric model allowed to verify significant relationships between training and IT abilities in the company and the success of the implementation, measured by the time required for this one. It was also verified the impact of this time over the perception of the companies global satisfaction with the ERP implementation. The investigation concludes with recommendations for its application and also suggests additional potential areas of investigation inspired by an amazing collateral result obtained.
\end{abstract}

Key words: ERP systems, integrated systems, enterprise resource planning, critical factors of success, success of the ERP in the small and medium company. 


\section{Introducción}

Los sistemas integrados de planificación de los recursos empresariales (ERP por sus siglas en inglés: enterprise resource planning) forman una tipología de TI que pretende integrar y automatizar los procesos medulares de las organizaciones, incluyendo finanzas, logística, ventas, procesamiento de órdenes, producción y planificación de materiales. Algunos estudios han estimado la inversión de las empresas en implementar los ERP en aproximadamente 300 billones de dólares estadounidenses en la década de 1990, y solo para el año 2004 se estimó que este valor estaría alrededor de los 79 billones de dólares (Carlino et ál., 2000), lo cual demuestra la relevancia actual del tema.

¿Por qué ha surgido, tanto en los académicos como en los empresarios, este creciente interés en analizar la implementación de los ERP? En lo esencial, por la presencia de experiencias contrapuestas: éxitos y fracasos. Cuando son implementados y asimilados con éxito, los beneficios generados por los ERP pueden ser muy relevantes, pues repercuten tanto en las operaciones como en los aspectos estratégicos del negocio (Shang y Seddon, 2000).

Algunos casos de estudio han revelado que la implementación exitosa de los ERP puede contribuir con ahorros en costos realmente notables gracias a la integración de procesos y la habilitación de formas innovadoras de conducir el negocio que impactan positivamente en la rentabilidad. También se ha documentado la repercusión directa de las mejoras debidas a los ERP sobre áreas medulares que inciden en la fuente de ingresos, como servicio al cliente, análisis de patrones de ventas y toma de decisiones en tiempo real (Davenport, 2000).
Hitt et ál. (2000) demostraron empíricamente que aquellas organizaciones que han invertido en ERP tienden a exhibir mejores desempeños financieros y mayor valoración en el mercado, en comparación con aquellas que no lo han hecho.

Si bien es cierto que en muchos casos los ERP potencian la ventaja competitiva de las organizaciones, en otros, su implementación ha generado fracasos dramáticos con deterioros irreversibles. Davenport (1998) presentó el caso de la compañía FoxMeyer Drugs que atribuyó a la implementación del ERP su posterior bancarrota. Ante estos resultados contradictorios, son múltiples las inquietudes que experimentan las empresas que evalúan la adopción de este tipo de tecnología en sus procesos de negocio (Davenport, 1998). Loh y Koh (2004) sostienen que la alta tasa de fracasos en la implementación de los ERP ofrece una oportunidad única a los investigadores para obtener un mejor entendimiento de aquellos elementos críticos que pueden determinar su éxito. Gargeya y Brady (2005) dan una aproximación a esta tasa de fracasos y sostienen que $70 \%$ de los proyectos ERP fallan en ser completamente implementados, inclusive después de 3 años invertidos en el intento.

Además, y aun cuando la implementación del ERP sea exitosa, no debe pasarse por alto que las empresas también enfrentan retos, obstáculos y riesgos previos al disfrute de sus beneficios. En términos de inversión, para las grandes empresas la adopción de un ERP puede requerir miles o incluso millones de dólares (Robey et ál., 2002). Otro factor, tan importante como la inversión, lo representa el tiempo de implementación del ERP, pues este compromete directamente tanto las operaciones del negocio como sus recursos. Los resultados 
obtenidos en este aspecto son también muy variados y, en algunos casos, profundamente desalentadores (Bajwa et ál., 2004).

\section{Propósito de la investigación}

Aun cuando los sistemas ERP fueron inicialmente destinados a abordar las necesidades de las grandes organizaciones, a partir del año 2000 se ha notado un sólido y sostenido crecimiento de su empleo en el sector de las pequeñas y las medianas empresas (pymes) (Van Everdingen et ál., 2000). Los riesgos y los obstáculos que enfrenta la implementación del ERP pueden parecer más impactantes si se considera el entorno de estas empresas, caracterizadas por estrictas limitaciones de conocimientos y recursos (Loh y Koh, 2004). Mabert et ál. (2000) sostienen que la inversión en ERP puede ser incluso más desafiante para este tipo de empresas, pues en algunos casos puede comprometer hasta la mitad de sus ingresos anuales, lo cual introduce un grado de vulnerabilidad superior para este tipo de organizaciones.

Si bien es cierto que la mayor parte de la investigación existente sobre ERP ha sido conducida para las grandes empresas (Loh y Koh, 2004), Mabert et ál. (2003) consideran que empresas de diversos tamaños pueden abordar los procesos de implementación del ERP de forma diferente pues les es posible establecer prioridades particulares.

La presente investigación empírica pretende contribuir al limitado conocimiento existente en materia de implementación de ERP en las pymes mediante el análisis de los factores que influyen en un resultado exitoso en estas empresas. En este sentido, se analizará el tiempo de implementación como una dimensión específica de su éxito.
Se tomará como punto de referencia los factores que impactan sobre ese tiempo de implementación como: a) gerencia de proyecto, $b$ ) adiestramiento $\mathrm{y} c$ ) habilidades en tecnología de la información (TI), los cuales ya han sido estudiados para el caso de las grandes empresas.

Estas son las preguntas que se intenta responder:

- ¿Existe relación entre la gerencia de proyecto, el adiestramiento y las capacidades de TI de la empresa con un tiempo de implementación exitoso en los proyectos de adopción de ERP en las pymes?

- ¿Existe relación entre el tiempo de implementación para la ejecución del ERP y la satisfacción con el proceso de implementación de este en las pymes?

En las siguientes secciones se abordarán la revisión de la literatura, la metodología de investigación aplicada, los resultados, la discusión relacionada, las limitaciones encontradas y las oportunidades de investigación adicionales que se derivan de estas.

\section{Revisión de la literatura}

\subsection{Los sistemas de planificación de los recursos empresariales (ERP)}

Los ERP son una suite de aplicaciones administrativas integradas que contempla soluciones de TI para los procesos de finanzas, contabilidad, recursos humanos, manufactura, logística, servicios y relaciones con los clientes. Su objetivo consiste en unir, bajo una plataforma única de información, todos los procesos del negocio de una empresa. 
La creciente y sostenida demanda de los ERP en el mercado empresarial resulta notable. Kalakota y Robinson (1999) precisan algunas necesidades fundamentales de las empresas como justificación de este interés:

- La necesidad de contar con una plataforma tecnológica que mejore el procesamiento de las órdenes de los clientes.

- La necesidad de consolidar y unificar las funciones del negocio, como manufactura, finanzas, distribución-logística $\mathrm{y}$ recursos humanos.

- La necesidad de integrar diversas tecnologías de información, junto con los procesos subyacentes que soportan, en una única «columna vertebral de información», en un «denominador común» informático que mejore la eficiencia.

- La necesidad de contar con una infraestructura informática que permita a las empresas adaptarse rápidamente al entorno actual de los negocios: disponer de capacidades como multiidiomas, multimonedas, multicompañías, etcétera.

Rowe (1999) describe la implementación de los sistemas ERP como la materialización del sueño de la gerencia empresarial de unificar y centralizar toda la infraestructura de sistemas de TI en un único sistema común. Los ERP proveen a los actores organizacionales de un lenguaje y un repositorio común de datos. En un nivel práctico, estos sistemas generan beneficios tangibles, por ejemplo:

- Unifican la infraestructura de tecnología de información.

- Estandarizan los procedimientos y los reportes operativos.
- Optimizan los procesos claves de la organización.

Además, ofrecen altos niveles de portabilidad y flexibilidad para adaptarse al entorno y los requerimientos específicos de las organizaciones (Rowe, 1999).

Compañías como Microsoft, Coca Cola, Cisco, Hershey Foods y Colgate $\mathrm{y}$, en América Latina, Telmex, PDVSA, Petrobras, Quilmes, Lan Chile, Wong y Federación de Cafeteros de Colombia han adoptado la tecnología ERP y han obtenido beneficios tangibles como reducción de inventario, disminución de costos operativos y mejora de la eficiencia en los procesos logísticos.

\subsection{Barreras en la implementación de los sistemas ERP}

La percepción de la alta inversión requerida, en términos de tiempo y costo, constituye una de las mayores preocupaciones de las organizaciones a la hora de emprender un proyecto de esta naturaleza. Inclusive, los sistemas ERP se ven como aptos para las grandes organizaciones que pueden permitirse invertir en el rediseño de los procesos medulares del negocio. Besson (1999) reportó cuán rápido los gerentes culpan a los sistemas ERP y su complejidad como la causa de proyectos fallidos.

Parece que cualquier subestimación por parte de la gerencia involucrada en estos casos sobre la escala del cambio organizacional requerido puede convertirse en una gran fuente de riesgo, muchas veces irreversible. 


\subsection{La experiencia de los sistemas ERP en las pymes}

Aun cuando los sistemas ERP fueron inicialmente destinados para las grandes organizaciones, es indudable que su implementación comienza a generar beneficios en organizaciones más pequeñas y con menos recursos financieros (Adam y O'Doherty, 2000). Fuerzas motoras como la globalización han puesto a las pymes en una nueva base de competencia y colaboración, por lo que resulta imperativo que incorporen tecnología de información que les permita abordar tales retos (Lebre La Rovere, 1996). Es evidente el impacto que los sistemas ERP pueden tener en este entorno.

Desde el punto de vista tecnológico, los motivos para la adopción de un sistema ERP en las pymes no son distintos de los de la gran empresa. Sus objetivos son similares: al adoptar un sistema ERP, las pymes buscan optimizar su desempeño organizacional mediante una mejor y más oportuna información, mayor control de la organización y el reemplazo de sistemas anticuados (Adam y O'Doherty, 2000; Thong, 1999).

\subsection{Tiempo de implementación en la ejecución del ERP}

Bajwa et ál. (2004) sostienen que el proceso de implementación del ERP compromete las operaciones normales del negocio y los recursos disponibles, por tanto, el tiempo de implementación es un factor crítico a considerar. En este sentido, los resultados son muy variados y en algunos casos desalentadores. Estos autores citan un estudio que reportó que $35 \%$ de las implementaciones de los ERP han sido canceladas, $55 \%$ han incurrido en excesos de tiempo y solo $10 \%$ han sido completadas dentro del tiempo previsto.
Hitt et ál. (2002) afirman que la implementación de los ERP es considerada como muy compleja y difícil. Para las grandes empresas, algunos estudios han estimado que percibir los beneficios tangibles puede tomar entre 1 y 3 años, en promedio, a partir de los 31 meses de efectuada (McAfee, 1999). Parte de las dificultades radica en los cambios asociados con el ERP, la necesidad de revaluar los procesos de las organizaciones y el nivel en el cual deben adaptarse los procesos a la capacidad del sistema. Esta complejidad induce diversos riesgos que potencialmente atentan contra el tiempo de implementación originalmente planificado. A su vez, el mayor tiempo en la implementación impacta sobre mayores costos asociados y, por ende, atenta directamente contra el nivel de satisfacción con el proceso. Este impacto debe repercutir, dadas las limitaciones mencionadas, con mayor intensidad en las pymes. De estos argumentos surge la siguiente hipótesis:

H1: En la implementación de los ERP en las pymes, el tiempo de implementación empleado y el nivel de satisfacción con dicha implementación se relacionan de manera negativa.

\subsection{Gerencia del proyecto durante la implementación del ERP}

Ahituv et ál. (2002) sostienen que la definición del ámbito del proyecto, una clara determinación de los recursos necesarios para el equipo que participa en este y un plan detallado son los aspectos fundamentales que se deben considerar en la fase de preparación de la gerencia del proyecto de implementación de un ERP. Holland et ál. (1999) y Shanks et ál. (2000) postulan que una definición precisa del alcance del 
proyecto plasmado en un plan de implementación claro debe ser el pilar de la gerencia de este y destacan la necesidad de trabajar sobre planes realistas. Boehm (1991) había anticipado también la importancia de contar con planes realistas dentro de la gerencia del proyecto como factor de éxito en cualquier intento de implementación de TI.

Bajwa et ál. (2004) sostienen que la implementación del ERP es extremadamente riesgosa debido a la amplitud de su ámbito, la naturaleza poco estructurada de sus tareas y la complejidad de la tecnología involucrada; como consecuencia, el empleo de herramientas de gerencia de proyecto apropiadas es absolutamente esencial para el éxito. Las herramientas de integración interna son críticas para coordinar las tareas del equipo de implementación y resolver los problemas técnicos que puedan surgir. Las herramientas de integración externa también son decisivas para facilitar la colaboración y asegurar que se tengan en cuenta los requerimientos de los usuarios finales. Las herramientas de planeación y control quizá puedan resultar menos útiles debido a la naturaleza propia y poco estructurada del proceso de implementación del ERP. Sin embargo, pueden ser necesarias para validar todas las especificaciones y todos los productos considerados, lo que permitirá evaluar el progreso del proyecto más adelante.

La implementación del ERP abarca todos los departamentos funcionales de la empresa, por lo que la cooperación entre técnicos y expertos de negocios es fundamental. Bingi et ál. (1999), Buckhout et ál. (1999) y Shanks et ál. (2000) sostienen inclusive que la mejor gente de la organización debería ser reclutada para el proyecto de implementación del ERP.
Acerca del líder del proyecto, Nah et ál. (2003) afirman que este papel es aún más importante, en comparación con el caso de cualquier otra tecnología de información, porque requiere de gran compromiso organizacional. Jiang et ál. (1996) indican que la designación de un gerente de proyecto competente es el segundo factor más importante para cualquier implementación de una TI. Estos autores han recopilado evidencia empírica que ratifica el papel del gerente del proyecto como factor crítico en la implementación de los proyectos de TI. También Nah et ál. (2003) señalan que la gerencia del proyecto es esencial en la implementación de los ERP pues es un factor que permitirá el logro de los objetivos en términos de tiempo y presupuesto.

Todos los argumentos anteriormente citados con referencia a la relación entre el nivel de gerencia del proyecto y el tiempo de implementación del ERP ejecutado se evidencian como especialmente relevantes para las pymes, por lo que se desprende la siguiente hipótesis:

H2: En la implementación del ERP en las pymes, el nivel de gerencia del proyecto aplicado y el tiempo de implementación empleado se relacionan en forma negativa.

\subsection{Adiestramiento durante la implementación del ERP}

Bingi et ál. (1999), Holland et ál. (1999), Roberts y Barrar (1992), Shanks et ál. (2000) y Stratman y Roth (2002) han destacado la importancia de suministrar adiestramiento formal a los usuarios finales como parte del proyecto de implementación del ERP. Este adiestramiento los ayudará a entender el sistema y también 
a comprender cómo sus tareas deben ser adaptadas y llevadas a cabo en lo sucesivo. Estos autores también indican que, por lo general, el adiestramiento es uno de los primeros rubros que se recorta cuando el proyecto comienza a exceder el presupuesto planificado, pero destacan que las empresas no debieran tomar esta acción que repercutirá frontalmente en los resultados posteriores de la implementación.

Sumner (1999) sostiene que, además del entrenamiento de los usuarios finales, es fundamental la capacitación en los temas técnicos propios de la tecnología del ERP y el adiestramiento para el equipo de implementación en temas de metodología y gerencia del proyecto.

Roberts y Barrar (1992) y Mainwaring (1999) afirman que el adiestramiento debería ser una actividad que se ejecute desde el inicio mismo del proyecto de implementación, pues a través de esta vía los involucrados podrán contribuir de forma más efectiva y eficiente a la adaptación de los procesos de negocios al sistema, acelerando así el proceso de implementación.

Todos estos argumentos sobre la relación entre el nivel de adiestramiento y el tiempo de implementación del ERP ejecutado parecen ser muy pertinentes para su aplicación en las pymes, por lo que se desprende la siguiente hipótesis:

H3: En la implementación del ERP en las pymes, el nivel de adiestramiento ejecutado y el tiempo de implementación empleado se relacionan negativamente.

\subsection{Habilidades de tecnología de información presentes en la empresa}

Bajwa et ál. (2004) sostienen que el grado de adaptación técnica que se haga al ERP durante su implementación tiene un efecto directo sobre su éxito. Una adaptación excesiva puede ser muy costosa y al mismo tiempo destruir la integridad del software e inclusive reducir los beneficios asociados. Estos autores también indican que las capacidades y las habilidades técnicas del equipo que lidera la implementación constituyen un factor fundamental. Asimismo, argumentan que, a diferencia de otras aplicaciones de TI, el ERP requiere de un equipo dedicado de soporte en la organización que permita controlar los aspectos de eficiencia técnica del sistema y atienda también a las inquietudes de los usuarios finales.

Nah et ál. (2003) afirman que las empresas deberían estar dispuestas a aceptar las mejores prácticas que vienen incluidas con el software y modelar sus procesos de negocio de acuerdo con estas prácticas para hacer más eficiente el proceso de implementación del ERP. Autores como Bingi et ál. (1999), Holland et ál. (1999), Murray y Coffin (2001) y Shanks et ál. (2000) sostienen que el ERP debería ser instalado con rapidez y modificado eficientemente, pues las empresas deberían estar dispuestas a adaptar sus procesos de negocio a las prácticas incorporadas en el ERP para explotar todas sus ventajas y ejecutar el proceso en el menor tiempo posible.

Markus et ál. (2000) destacan, dentro de los temas propios de TI involucrados en la implementación del ERP, la importancia de la depuración de los datos y de una migración ordenada de estos al nuevo sistema. Estas tareas relacionadas con la calidad de 
los datos existentes pueden ser subestimadas y merecen una atención especial con la finalidad de evitar retrasos dramáticos en el proceso de implementación.

Los argumentos anteriores sobre la relación entre el nivel de habilidades en materia de TI existente en la empresa y el tiempo de implementación del ERP son especialmente aplicables para el caso de las pymes, por lo que se desprende la siguiente hipótesis:

H4: En la implementación del ERP en las pymes, el nivel de habilidades de tecnología de la información presentes en la empresa y el tiempo de implementación empleado están negativamente relacionados.

El modelo de investigación propuesto en la presente investigación se resume en el gráfico 1.
El cuadro 1 presenta un resumen de las variables involucradas y su relevancia en la literatura.

\section{Metodología}

La presente investigación adoptó una perspectiva empírica y cuantitativa orientada a verificar hipótesis y, por ende, las relaciones propuestas entre variables. Se siguió una estrategia de investigación tipo encuesta (survey). Los datos se recopilaron mediante una muestra de pymes a la que se aplicó una encuesta en un lapso de tiempo determinado (estudio cross-sectional). Se diseñó un cuestionario para facilitar la recolección de información de la muestra seleccionada.

La unidad de análisis de esta investigación es la empresa.

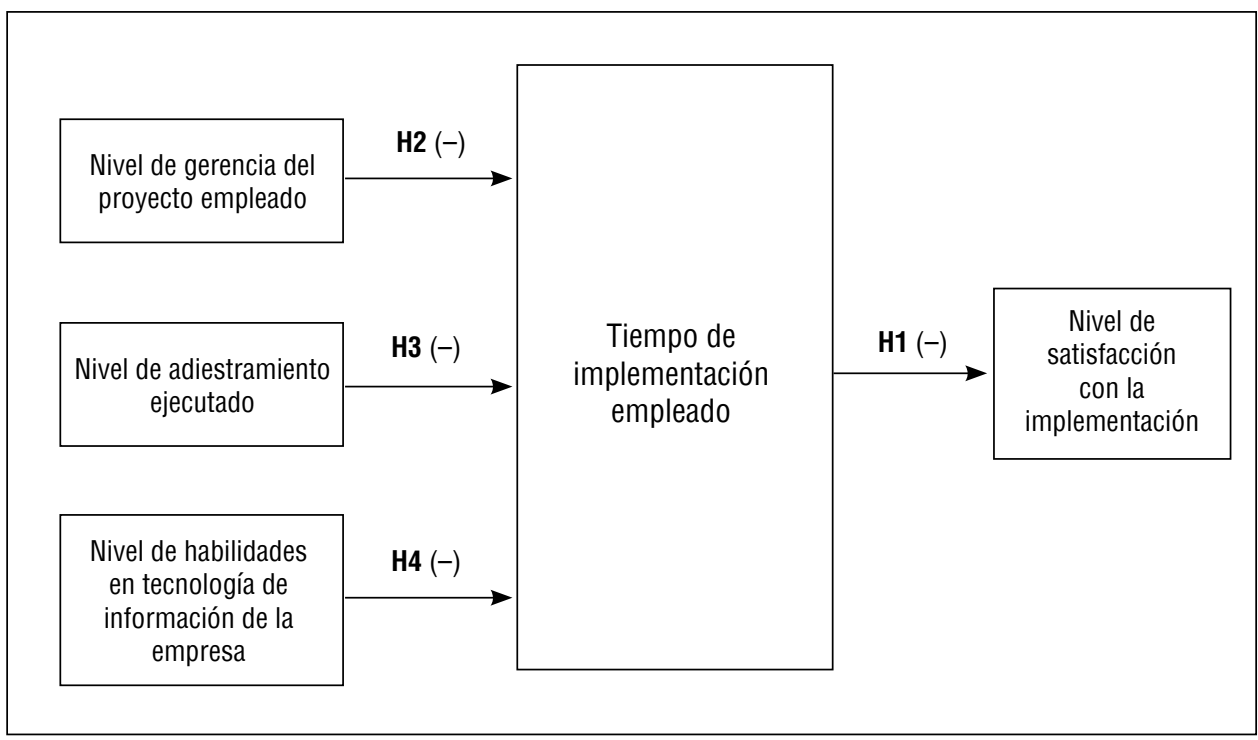

Gráfico 1

Modelo de investigación 


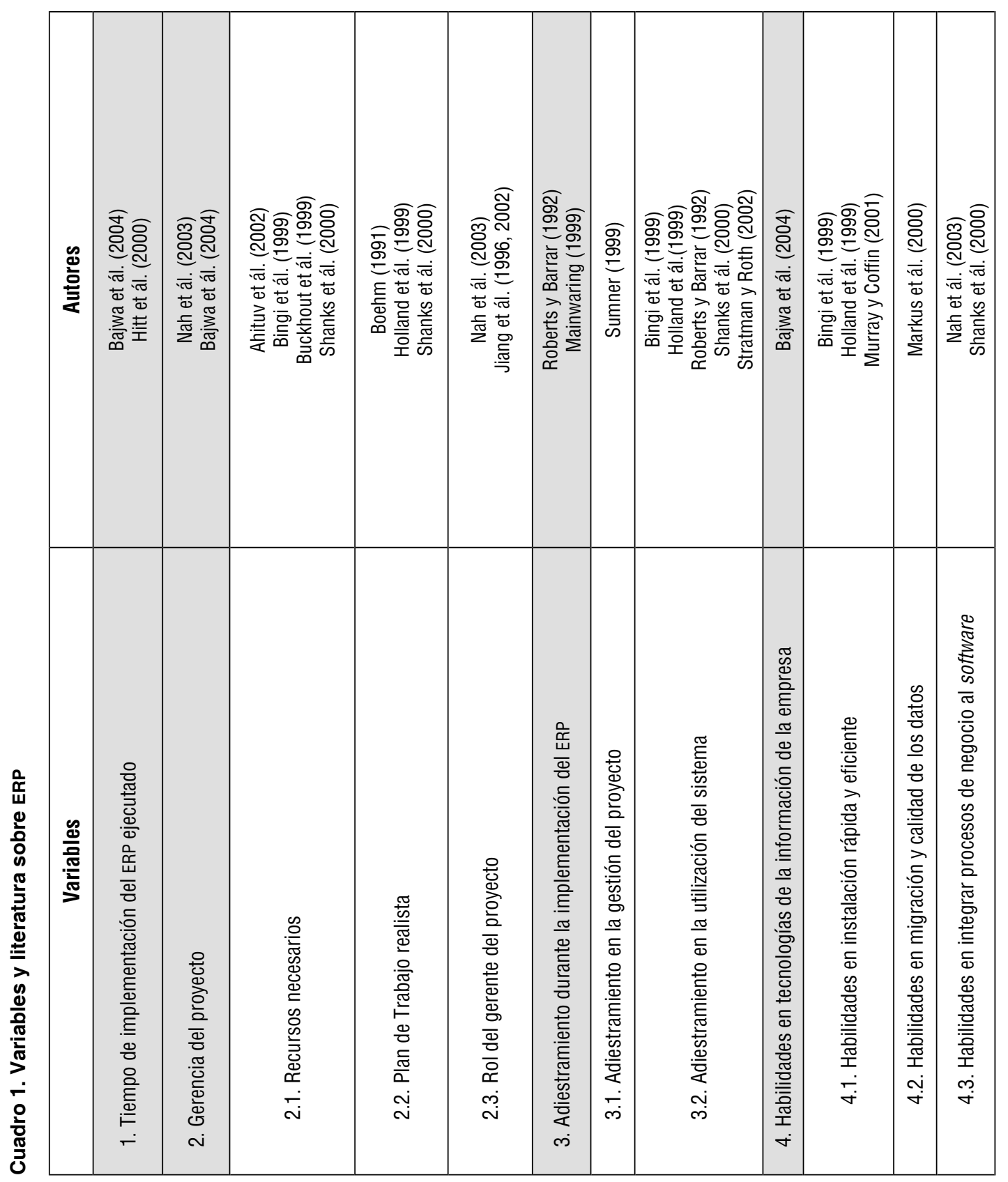




\subsection{Descripción de la muestra}

El universo seleccionado pertenece al conjunto de pymes que han implementado un ERP de un determinado proveedor líder en América Latina. Se debe destacar que la definición de pymes de este proveedor coincide con las características especificadas por Zevallos (2003) y Boggs et ál. (2007), quienes las definen como empresas que constan de hasta 500 empleados.

Esta lista original tenía 52 empresas que habían implementado ese determinado ERP y estaban ubicadas en diferentes países de América Latina, incluyendo México, Brasil, Guatemala, Costa Rica, Perú y algunos del Caribe. Se seleccionó de forma aleatoria una muestra de 40 empresas. Para hacerlo, se asignó un número de identificación a cada una de las empresas (comprendido entre 1 y 40) y se generaron 40 números al azar, mediante el uso de una rutina computarizada. Los números generados indicaron la identificación de las empresas que fueron invitadas a participar.

La invitación a participar en el estudio fue enviada al dueño o director general de la organización a través de un correo electrónico en el cual se destacaba la naturaleza voluntaria, el objetivo del estudio, sus beneficios y el compromiso de confidencialidad de la información. De las 40 empresas, 34 accedieron a participar en el estudio y respondieron el cuestionario enviado. Debido a la existencia de cuestionarios con información incompleta en algunos tópicos, la muestra final abarcó 15 empresas, con lo que se obtuvo una tasa final de participación de $38 \%$.

Las organizaciones que no participaron no diferían en número de empleados respecto de las que sí lo hicieron.

\subsection{Operacionalización de las variables}

Las variables consideradas fueron: tiempo de implementación del ERP, nivel de satisfacción con su implementación, nivel de gerencia durante esta, nivel de adiestramiento en el proceso y habilidades de tecnología de la información presentes en la empresa.

\section{Tiempo de implementación empleado en el ERP}

Inicialmente se planteó utilizar como medida de esta variable la duración en semanas del tiempo de implementación; pero, ya que las implementaciones de un ERP pueden variar en las diferentes empresas de acuerdo con los objetivos establecidos (procesos de negocio que deben contemplarse en la implementación), se decidió que esta medición podría originar confusiones en la interpretación y no necesariamente permitiría establecer comparaciones equivalentes entre organizaciones, pues no todas las empresas deciden implementar todos y los mismos procesos de negocio en el ERP.

Se optó entonces por utilizar una variable con una escala tipo semántica diferencial (Babbie, 1990), donde se preguntaba sobre el resultado en «tiempo de implementación» empleado con respecto de las expectativas iniciales. Los participantes respondieron según una escala que iba de 1 («El tiempo de implementación fue muy por debajo a lo esperado») a 5 («El tiempo de implementación fue muy excedido a lo esperado»). Esta alternativa coincide con la utilizada por Reinartz et ál. (2004) desde la perspectiva del uso de una medida perceptual del constructo. 
Nivel de satisfacción con la implementación del ERP

También en este caso se empleó una variable con una escala tipo semántica diferencial (Babbie, 1990), en la cual se preguntaba sobre el nivel de «satisfacción general» con la implementación del ERP en la empresa. Los participantes respondieron de acuerdo con una escala que iba de 1 («Nada satisfecho con la implementación del ERP») a 5 («Muy satisfecho con la implementación del ERP»). Esta alternativa coincide con la usada por Reinartz et ál. (2004) en la perspectiva de una medida perceptual del constructo.

\section{Nivel de gerencia del proyecto durante la implementación del ERP}

Se utilizó una medida de tres variables basada en Nah et ál. (2003) con una escala tipo semántica diferencial (Babbie, 1990) que va de 1 («Nada satisfecho») a 5 («Muy satisfecho»):

- Claro establecimiento de los recursos necesarios para la implementación

- Definición de un Plan de Trabajo realista

- Establecimiento del líder del proyecto

Estos tres elementos mostraron una buena confiabilidad: $\alpha=0,92$ (ver reporte estadístico detallado en el anexo 1); luego se promediaron para generar el factor correspondiente.

\section{Nivel de adiestramiento durante la implementación del ERP}

Se utilizó una medida de dos variables basada en Nah et ál. (2003) y Holland et ál. (1999) con una escala tipo semántica diferencial (Babbie, 1990) que iba desde
1 («Nada satisfecho») hasta 5 («Muy satisfecho»):

- Capacitación adecuada y suficiente durante la administración del proyecto

- Capacitación adecuada y suficiente en la utilización del software ERP

Estos dos aspectos presentaron una buena confiabilidad: $\alpha=0,70$ (ver reporte estadístico detallado en el anexo 1); luego se promediaron para generar el factor correspondiente.

Habilidades de tecnología de la información presentes en la empresa

Se utilizó una medida de variables basada en Nah et ál. (2003) y Markus et ál. (2000) con una escala tipo semántica diferencial (Babbie, 1990) que iba desde 1 («Nada satisfecho») hasta 5 («Muy satisfecho»):

- Instalación e inicio rápido y eficiente del software ERP

- Migración ordenada de los datos al software ERP

- Integración eficiente de los procesos de negocio al software ERP

Estos aspectos mostraron una relativa confiabilidad: $\alpha=0,52$ (ver reporte estadístico detallado en el anexo 1); luego se promediaron para generar el factor correspondiente.

Se debe destacar que se verificó la «validez discriminante» de las variables y las medidas anteriores mediante la ejecución de un análisis factorial con el empleo específico de la técnica de «Extracción de análisis de componentes principales» con el método de rotación Varimax y normalización Kaiser (ver reporte estadístico detallado en el anexo 1). 
El cuestionario final empleado, basado en las definiciones descritas anteriormente y diseñado considerando las guías metodológicas suministradas por Roberts (1999) se presenta en el anexo 4.

\section{Resultados}

A continuación se presenta el perfil demográfico de las empresas entrevistadas. Así, $62 \%$ de las empresas entrevistadas cuenta con menos de 100 empleados y $31 \%$ tiene menos de 50 empleados. La empresa entrevistada con menos empleados tiene únicamente 12. En cuanto a las ventas anuales, el promedio es de 9 millones de dólares anuales. La empresa con menores ingresos vende alrededor de 130 mil dólares anuales. Respecto de los años en el mercado, el promedio de estos es 15 años. La empresa con menos tiempo en el mercado tiene 3 años y la más antigua, 58 años.

En cuanto a los sectores a los cuales pertenecen las empresas, $37 \%$ se ubica en el sector manufactura; $25 \%$, en el sector servicios; y $38 \%$, en el sector de ventas minoristas (cuadro 2).

El cuadro 3 muestra un análisis de la correlación de las variables utilizadas.

Se utilizará el análisis de regresión lineal múltiple para verificar las hipótesis. Hair et ál. (2005) sostienen que el análisis de regresión múltiple es la técnica estadística apropiada cuando el problema de investigación pretende analizar una presunta relación entre una variable dependiente $\mathrm{y}$ varias variables independientes.

El cuadro 4 presenta los resultados del análisis de regresión cuando la variable dependiente es tiempo de implementación empleado y las variables independientes son nivel de gerencia del proyecto, nivel de adiestramiento ejecutado y nivel de habilidades en TI de la empresa (el anexo 2 presenta en forma exhaustiva los resultados de la regresión y el cumplimiento de las hipótesis subyacentes).

\section{Cuadro 2. Análisis descriptivo de las variables utilizadas}

\begin{tabular}{|c|c|c|c|c|c|}
\hline Variable & Significado & Promedio & $\begin{array}{c}\text { Desviación } \\
\text { estándar }\end{array}$ & Mínimo & Máximo \\
\hline FACPROMGM & $\begin{array}{c}\text { Nivel de gerencia } \\
\text { del proyecto }\end{array}$ & 3,8888889 & 0,8967903 & 2,0000000 & 5,0000000 \\
\hline FACTRAINING & Adiestramiento & 3,3666667 & 1,0082989 & 1,0000000 & 4,5000000 \\
\hline FACIT & $\begin{array}{c}\text { Habilidades de } \\
\text { tecnología } \\
\text { de la información }\end{array}$ & 3,6000000 & 0,7580258 & 1,6666667 & 5,0000000 \\
\hline SATISFIMPLEMENT & $\begin{array}{c}\text { Nivel de } \\
\text { satisfacción con } \\
\text { la implementación }\end{array}$ & 3,2500000 & 0,9309493 & 1,0000000 & 4,0000000 \\
\hline RESULTADOTIEMPO & $\begin{array}{c}\text { Tiempo de } \\
\text { implementación } \\
\text { empleado }\end{array}$ & 3,6875000 & 0,9464847 & 3,0000000 & 5,0000000 \\
\hline
\end{tabular}


Cuadro 3. Análisis de la correlación de las variables

\begin{tabular}{|c|c|c|c|c|}
\hline & \multicolumn{3}{|c|}{$\begin{array}{c}\text { Pearson correlation coefficients } \\
\text { Prob }>|r| \text { under H0: Rho }=0 \\
\text { Number of observations }\end{array}$} & \multirow[b]{2}{*}{ RESULTADOTIEMPO } \\
\hline & FACPROMGM & FACTRAINING & FACIT & \\
\hline $\begin{array}{l}\text { FACPROMGM } \\
\text { FACPROMGM }\end{array}$ & $\begin{array}{r}1,00000 \\
15\end{array}$ & $\begin{array}{r}0,70655 \\
0,0032 \\
15\end{array}$ & $\begin{array}{r}0,57207 \\
0,0259 \\
15\end{array}$ & $\begin{array}{r}-0,17500 \\
0,5327 \\
15\end{array}$ \\
\hline $\begin{array}{l}\text { FACTRAINING } \\
\text { FACTRAINING }\end{array}$ & $\begin{array}{r}0,70655 \\
0,0032 \\
15\end{array}$ & $\begin{array}{r}1,00000 \\
15\end{array}$ & $\begin{array}{r}0,42366 \\
0,1156 \\
15\end{array}$ & $\begin{array}{r}-0,49030 \\
0,0635 \\
15\end{array}$ \\
\hline $\begin{array}{l}\text { FACIT } \\
\text { FACIT }\end{array}$ & $\begin{array}{r}0,57207 \\
0,0259 \\
15\end{array}$ & $\begin{array}{r}0,42366 \\
0,1156 \\
15\end{array}$ & $\begin{array}{r}1,00000 \\
15\end{array}$ & $\begin{array}{r}-0,48999 \\
0,0637 \\
15\end{array}$ \\
\hline $\begin{array}{l}\text { RESULTADOTIEMPO } \\
\text { RESULTADOTIEMPO }\end{array}$ & $\begin{array}{r}-0,17500 \\
0,5327 \\
15\end{array}$ & $\begin{array}{r}-0,49030 \\
0,0635 \\
15\end{array}$ & $\begin{array}{r}-0,48999 \\
0,0637 \\
15\end{array}$ & $\begin{array}{r}1,00000 \\
16\end{array}$ \\
\hline
\end{tabular}

Cuadro 4. Resultados del análisis de regresión de la variable dependiente tiempo de implementación empleado y de las variables independientes gerencia, adiestramiento y habilidades en $\mathrm{TI}$

\begin{tabular}{|c|c|c|c|c|c|c|c|c|}
\hline \multicolumn{9}{|c|}{ Parameter estimates } \\
\hline Variable & Label & DF & $\begin{array}{l}\text { Parameter } \\
\text { estimate }\end{array}$ & $\begin{array}{c}\text { Standard } \\
\text { error }\end{array}$ & $t$ value & $\operatorname{Pr}>|t|$ & Tolerance & $\begin{array}{l}\text { Variance } \\
\text { inflation }\end{array}$ \\
\hline Intercept & Intercept & 1 & 5,62287 & 1,00799 & 5,58 & 0,0002 & & 0 \\
\hline FACTPROMGM & FACTPROMGM & 1 & 0,65645 & 0,33484 & 1,96 & 0,0758 & 0,41013 & 2,43827 \\
\hline FACTRAINING & FACTRAINING & 1 & $-0,64135$ & 0,26966 & $-2,38$ & 0,0366 & 0,50022 & 1,99912 \\
\hline FACIT & FACIT & 1 & $-0,67126$ & 0,30948 & $-2,17$ & 0,0529 & 0,67198 & 1,48814 \\
\hline
\end{tabular}

Nota: $R^{2}=0,50, p<0,05$

Con respecto de nivel de adiestramiento ejecutado, los resultados corroboran la hipótesis 3, pues se verifica su relación negativa con el tiempo de implementación empleado: $\beta=-0,64135, \mathrm{p}<0,05$.

En cuanto a nivel de habilidades en tecnología de la información de la empresa, los resultados corroboran la hipótesis 4, pues se verifica una relación negativa con el tiempo de implementación empleado: $\beta=-0,67126, \mathrm{p}<0,10$.

Finalmente, respecto de nivel de gerencia de proyecto empleado, sorprendentemente, y en contra a lo que se planteó en la hipótesis 2 , se verificó una relación positiva con el tiempo de implementación empleado: $\beta=0,65645, \mathrm{p}<0,10$. En este sentido, la hipótesis no fue corroborada. 
El cuadro 5 reporta los resultados del análisis de regresión cuando la variable dependiente es nivel de satisfacción con la implementación del ERP y la variable independiente es tiempo de implementación empleado. Los resultados obtenidos corroboran la hipótesis 1 , pues se verifica la relación negativa entre estas variables: $\beta=-0,51724, \mathrm{p}<0,05)$ (el anexo 3 presenta en detalle los resultados de la regresión y el cumplimiento de las hipótesis subyacentes).

El cuadro 6 resume las hipótesis involucradas y los resultados obtenidos.

\section{Resultados, limitaciones y perspectivas}

En este acápite final se presentan los resultados, las limitaciones, sus implicancias para las empresas y las contribuciones y las perspectivas de esta investigación.

\subsection{Resultados}

La presente investigación contribuye con el conocimiento de los factores que pueden determinar el éxito en la implementación del ERP, especialmente en el área

Cuadro 5. Análisis de regresión de nivel de satisfacción con la implementación del ERP como variable dependiente y tiempo de implementación empleado como variable independiente

Parameter estimates

\begin{tabular}{|llc|cccccc|}
\hline Variable & Label & DF & $\begin{array}{c}\text { Parameter } \\
\text { estimate }\end{array}$ & $\begin{array}{c}\text { Standard } \\
\text { error }\end{array}$ & $\mathbf{t}$ value & Pr $>|\mathbf{t}|$ & Tolerance & $\begin{array}{r}\text { Variance } \\
\text { inflation }\end{array}$ \\
\hline Intercept & Intercept & 1 & 5,19540 & 0,86573 & 6,00 & $<, 0001$ & 0 \\
RESULTADOTIEMPO & RESULTADOTIEMPO & 1 & 0,51724 & 0,23361 & $-2,21$ & 0,0453 & 1,00000 & 1,00000 \\
\hline
\end{tabular}

Nota: $R^{2}=0,27, p<0,05$

Cuadro 6. Hipótesis involucradas y sus resultados

\begin{tabular}{|c|c|c|c|c|}
\hline Hipótesis & Variable dependiente & Variable independiente & $\begin{array}{c}\text { Sentido de la } \\
\text { relación }\end{array}$ & ¿Corroborada? \\
\hline H1 & $\begin{array}{c}\text { Nivel de satisfacción con la } \\
\text { implementación del ERP }\end{array}$ & $\begin{array}{c}\text { Tiempo de implementación } \\
\text { empleado }\end{array}$ & Negativo & Sí \\
\hline H2 & $\begin{array}{c}\text { Tiempo de implementación } \\
\text { empleado }\end{array}$ & $\begin{array}{c}\text { Nivel de gerencia del } \\
\text { proyecto empleado }\end{array}$ & Negativo & $\begin{array}{c}\text { Resulta significativa } \\
\text { una relación en } \\
\text { sentido contrario } \\
\text { (positivo) }\end{array}$ \\
\hline H3 & $\begin{array}{c}\text { Tiempo de implementación } \\
\text { empleado }\end{array}$ & $\begin{array}{c}\text { Nivel de adiestramiento } \\
\text { ejecutado }\end{array}$ & Negativo & Sí \\
\hline H4 & $\begin{array}{c}\text { Tiempo de implementación } \\
\text { empleado }\end{array}$ & $\begin{array}{r}\text { Nivel de habilidades en TI de } \\
\text { la empresa }\end{array}$ & Negativo & Sí \\
\hline
\end{tabular}


de las pymes. Se concentró el interés en estudiar las variables que pueden incidir directamente en el tiempo de implementación empleado ${ }^{1}$, el cual es una dimensión fundamental que todas las empresas, en especial las pymes, por sus especiales limitaciones, deben comprender y controlar (Shehab et ál., 2004).

Los resultados obtenidos corroboran la importancia de que las pymes cuenten con sólidas habilidades en materia de tecnología de la información a la hora de emprender un proyecto de implementación de ERP. Si bien es cierto que este tipo de proyectos requiere de capacidades estratégicas y de negocios, no deben descuidarse las capacidades en materia de TI que pueden marcar la diferencia (Holland et ál., 1999; Markus et ál., 2000). De no contar una pyme con el conocimiento técnico requerido, será necesario emplear un tiempo adicional en el reclutamiento, el adiestramiento y la puesta en marcha para disponer de estas habilidades, lo que inevitablemente requerirá de un mayor tiempo para activar el ERP.

Con respecto del nivel de adiestramiento, se confirma la importancia de ejecutar un proceso de transferencia de conocimientos desde el inicio mismo de la implementación, tanto al equipo del proyecto como a los usuarios involucrados. Esto facilita la asimilación eficaz no solo de las capacidades y las limitaciones del sistema ERP, sino también de alternativas eficientes para adaptar los procesos de negocio a la nueva plataforma. Un conocimiento temprano de las características y las funciones del sistema permitirá crear alternativas ágiles cuando surjan obstáculos en el proceso de implementación, lo que puede permitir acortar el tiempo necesario para todo el proceso.

1. Siempre tomando como referencia las expectativas iniciales.
En cuanto al nivel de gerencia del proyecto, resultó sorpresiva la relación positiva y significativa obtenida con el tiempo de implementación empleado, en contra del sentido de la hipótesis propuesta. Una posible explicación para este hallazgo puede estar relacionada con lo señalado por Yen et ál. (2002), quienes indican que, por lo general, las pymes no están acostumbradas a enfrentar proyectos de gran envergadura. En la mayor parte de los casos no están adiestradas en el uso metodologías y revisiones minuciosas de los procesos de negocio. Tampoco es usual que cuenten con documentación sobre tales procesos. En estos casos, para cumplir con la metodología estricta que puede requerir la gerencia del proyecto, puede ser necesario mucho trabajo adicional para analizar y documentar los procesos de negocio que deben implementarse. Esto indefectiblemente impacta en el tiempo requerido para la implementación.

Por otra parte, una gerencia de proyectos disciplinada debe garantizar que el equipo del proyecto siempre esté enfocado en el proceso. Dadas las limitaciones de recursos que experimentan las pymes, es usual que un mismo empleado desempeñe varios roles pues los empleados suelen compartir sus labores tradicionales con el proceso de implementación del ERP. Es muy probable que en muchos casos el día a día absorba el tiempo que los empleados deberían disponer para el proyecto y, en este sentido, una gerencia de proyecto disciplinada no culminará las tareas correspondientes hasta contar con el apoyo y la validación de los recursos respectivos; lo que ocasionará retrasos en el tiempo de implementación. Marsh (2000) resume esta perspectiva y argumenta que la poca experiencia de la empresa en afrontar proyectos de escala similar en TI puede impactar sobre su proceso de implementación. 
Finalmente, se verifica también que, para las pymes, el tiempo de implementación empleado ${ }^{2}$ incide en la satisfacción general con el proceso, tal como fue indicado por Zviran et ál. (2005). Considerando las limitaciones que enfrentan las pymes, de acuerdo con Yen et ál. (2002), se desprende que una implementación de ERP que involucre menores tiempos a los esperados definitivamente supondrá un mayor nivel de satisfacción.

\subsection{Limitaciones}

Existen razones para ser cautelosos con la generalización de los resultados de la presente investigación. Primero, porque las empresas analizadas se ubican en el contexto latinoamericano. Los hallazgos aquí encontrados pudieran no ser aplicables a otras latitudes. Segundo, la muestra de las empresas que respondieron el cuestionario es limitada y la generalización y la potencia estadística subyacente pudieran verse cuestionadas, según lo señalado por Baroudi y Orlikowski (1989). Tercero, el éxito de la implementación del ERP se midió principalmente considerando el tiempo de implementación con respecto a las expectativas iniciales; sin embargo, otras dimensiones importantes del éxito quedaron fuera del alcance de esta investigación. Cuarto, la muestra se focalizó en pymes que habían implementado el ERP de un proveedor en particular, pues no se consideraron empresas que habían utilizado otros proveedores.

Una limitación adicional que se detectó es aquella relacionada con los constructos y las mediciones involucradas. No se encontraron prácticamente referencias de

2. Siempre tomando como referencia las expectativas iniciales. trabajos empíricos previos, en línea con lo mencionado por autores como Kumar et ál. (2002), quienes sostienen que existe poca investigación empírica relacionada con los principales obstáculos para la adopción de los ERP en las organizaciones. En este sentido, las mediciones propuestas están sujetas a mejoras y ampliaciones.

\subsection{Implicancias para las empresas}

A pesar de las limitaciones indicadas, el estudio ofrece importantes lecciones que deben ser consideradas por las pymes que están evaluando la posibilidad de implementar un ERP. En primer lugar, debe darse total prioridad desde el inicio al adiestramiento de los miembros del equipo del proyecto, tanto en los temas de gerencia del proyecto, como en aquellos propios del conocimiento del sistema de ERP. Dadas las restricciones de las pymes, existe la tendencia a minimizar el presupuesto $\mathrm{y}$, por lo general, el adiestramiento es una de las líneas susceptibles de reducción. El presente estudio contribuye a reforzar la importancia del adiestramiento y el riesgo que puede ocasionar, tanto en tiempo requerido como en posterior satisfacción, cualquier reducción en esta materia. ¡No escatime en adiestramiento!

También es muy importante que las pymes reflexionen sobre las capacidades existentes, «puertas adentro», en materia de TI. Debe existir la certeza de que se cuenta con un inventario de estas habilidades, lo que facilitará el proceso de implementación. De no disponer de tales habilidades, los hallazgos del presente estudio sugieren que la pyme evalúe con prudencia la postergación del proceso de implementación. 


\subsection{Contribuciones y futuras áreas de investigación}

Tal como afirma Hitt (2002), la mayor parte de la investigación sobre los ERP ha sido llevada a cabo principalmente a través de estudios de caso y encuestas en industrias y, además, la investigación existente ha estado orientada al segmento de grandes empresas y corporaciones (Shehab et ál., 2004).

El presente estudio pretende aportar nuevas luces considerando tales brechas y por eso se concentró en el análisis de las pymes, con una investigación empírica a través de encuestas a empresas pertenecientes a diversas industrias e inclusive en diferentes países; este hecho tiene mayor relevancia si se considera que en muchos de ellos las pymes constituyen el motor de la economía (Shehab et ál., 2004). América Latina no escapa a esta realidad y, en ese sentido, este estudio también contribuye en esa dirección, dado que no se encontraron investigaciones que abordaran la realidad latinoamericana.
Shehab et ál. (2004) sostienen que pueden existir diferencias en la implementación del ERP por parte de las pymes frente a las grandes empresas. Inclusive este hecho motiva los estudios en esta dirección. El hallazgo encontrado en esta investigación relacionado con el tópico gerencia del proyecto puede ser un ejemplo de estas diferencias y, en este sentido, apoya la invitación de Shehab et ál. (2004) de seguir profundizando en el segmento de las pymes para consolidar un mejor entendimiento y poder establecer comparaciones pertinentes con las grandes empresas.

Este estudio se concentró en el análisis del tiempo de implementación ejecutado en comparación con las expectativas iniciales como una de las dimensiones del éxito del proyecto. Definitivamente, existen otras dimensiones que contribuyen al éxito, cuya investigación debe ser profundizada en el futuro. Establecer los constructos subyacentes de tales dimensiones sería un aporte fundamental para el desarrollo de investigaciones sucesivas. 


\section{Referencias bibliográficas}

ADAM, F. y O'DOHERTY, P. 2000. Lessons from enterprise resource planning implementation in Ireland: Towards smaller and shorter ERP projects. Journal of Information Technology. N. ${ }^{\circ} 15$, págs. 305-316.

AHITUV, N.; NEUMANN, S. y ZVIRAN, M. 2002. A system development methodology for ERP systems. Journal of Computer Information Systems. Vol. 42, n. ${ }^{\circ}$ 3, págs. 56-67.

BABBIE, E. 1990. Survey research methods. Belmont, CA: Wadsworth.

BAJWA, D.; GARCIA, J. y MOONEY, T. 2004. An integrative framework for the assimilation of enterprise resource planning systems: Phases, antecedents, and outcomes. Journal of Computer Information Systems. Spring, págs. 81-90.

BAROUDI, J. y ORLIKOWSKI, W. 1989. The power of statistical power in MIS research. MIS Quarterly. March, págs. 87-106.

BESSON, P. 1999. Les ERP á l'épreuve de 1'organisation. Systémes d'information et management. Vol. 4, n. ${ }^{\circ}$, págs. 21-52.

BINGI, P.; SHARMA, M. y GODLA, J. 1999. Critical issues affecting an ERP implementation. Information Systems Management. Vol. 16, n. ${ }^{\circ}$ 2, págs. 7-14.

BOEHM, B. 1991. Software risk management: Principles and practices. IEEE Software. N. ${ }^{\circ} 8$, págs. $32-41$.

BOGGS, R.; CONVERSO, L.; KOCH, G.; GIBIN, E.; LEE, W. y SANDLER, M. 2007. Worldwide SMB 2007 Top 10 predictions: Market position index and growth opportunity ratings by region. IDC Document N. ${ }^{\circ}$ 205251, págs. 1-12.
BUCKHOUT, S.; FREY, E. y NEMEC, J. 1999. Making ERP succeed: Turning fear into promise. IEEE Engineering Management Review. ‥ ${ }^{\circ} 19$, págs. 116-123.

CARLINO, J.; NELSON, S. y SMITH, N. 2000. AMR research predicts enterprise applications market will reach \$78 billions by 2004. Boston, MASS: AMR Research.

DAVENPORT, T. 2000. Mission critical. Boston, MASS: Harvard Business School Press.

1998. Putting the enterprise in the enterprise system. Harvard Business Review. N. ${ }^{\circ}$ 76, págs. 121-131.

FIELD, A. 2005. Discovering statistics using SPSS. London: Sage.

GARGEYA, V. y BRADY, C. 2005. Success and failure factors of adopting SAP in ERP system implementation. Business Process Management. Vol. 11, n. ${ }^{\circ}$, págs. 501-518.

HAIR, J.; BLACK, W.; BABIN, B.; ANDERSON, R. y TATHAM, R. 2005. Multivariate data analysis. 6th edition. New Jersey, NJ: Pearson / Prentice Hall.

HITT, L.; WU, D. y ZHOU, X. 2002. Investment in enterprise resource planning: Business impact and productivity measures. Journal of Management Information Systems. Vol. 19, n. ${ }^{\circ} 1$, págs. 71-98.

HOLLAND, C.; LIGHT, B. y GIBSON, N. 1999. A critical success factors model for enterprise resource planning implementation. Proceedings of the Seventh European Conference on Information Systems. Copenhague.

JIANG, J.; KLEIN, G. y BALLOUN, J. 1996. Ranking of system implementation success 
factors. Project Management Journal. N. ${ }^{\circ} 27$, págs. $49-53$.

y DISCENZA, R. 2002. Pre-project partnering impact on information system project, project team and project manager. European Journal of Information Systems. N. ${ }^{\circ} 11$, págs. 86-97.

KALAKOTA, R. y ROBINSON, M. 1999. E-business: Roadmap to success. Reading: Addison-Wesley.

KUMAR, V.; MAHESHWARI, B. y KUMAR, U. 2002. Enterprise resource planning systems adoption process: A survey of Canadian organizations. International Journal of Production Research. Vol. 40, n. ${ }^{\circ}$ 3, págs. 509-523.

LEBRE LA ROVERE, R. 1996. IT diffusion in small and medium-sized enterprises: Elements for policy definition. Information Technology for Development. ‥ ${ }^{\circ}$, págs. 169-181.

LOH, T. y KOH, C. 2004. Critical elements for a successful enterprise resource planning implementation in small- and mediumsized enterprises. International Journal of Production Research. Vol. 42, n. ${ }^{\circ}$ 17, págs. 3433-3455.

MABERT, V.; SONI, A. y VENKATARAMANAN, M. 2003. The impact of organisation size on enterprise resource planning (ERP) implementations in the US manufacturing sector. Omega. N. ${ }^{\circ} 31$, págs. 235-246.

2000. Enterprise resource planning survey of US manufacturing firms. Production and Inventory Management Journal. Vol. 41, n. ${ }^{\circ} 2$, págs. 52-58.

MAINWARING, J. 1999. Training: The key to ERP implementation. Manufacturing Computer Solutions. N. ${ }^{\circ}$ 5, págs. 36-37.
MARKUS, M.; AXLINE, S.; PETRIE, D. y TANIS, C. 2000. Learning from adopters' experiences with ERP: Problems encountered and success achieved. Journal of Information Technology. ․ ${ }^{\circ} 15$, págs. 245-265.

MARSH, A. 2000. The implementation of enterprise resource-planning systems in small-medium manufacturing enterprises in South-East Queensland: A case study approach. Proceedings of the 2000 IEEE International Conference on Management Innovation and Technology. Singapure.

McAFEE, A. 1999. The impact of enterprise resource planning systems on company performance. Philadelphia, PENN: Wharton Electronic Supply Chain Conference.

MURRAY, M. y COFFIN, G. 2001. A case study analysis of factors for success in ERP System implementations. Boston, MASS: Proceedings of the 7th Americas Conference on Information Systems.

NAH, F.; ZUCKWEILER, K. y LAU, J. 2003. ERP implementation: Chief information officers' perceptions of critical success factors. International Journal of Human-Computer Interactions. Vol. 16, n. ${ }^{\circ}$ 1, págs. 5-22.

REINARTZ, W.; KRAFFT, M. y HOYER, W. 2004. The customer relationship management process: Its measurement and impact on performance. Journal of Marketing Research. Vol. XLI, págs. 293-305.

ROBERTS, E. 1999. In defense of the survey method: An illustration from a study of user information satisfaction. Accounting and Finance. N. ${ }^{\circ} 39$, págs. 53-77.

ROBERTS, H. y BARRAR, P. 1992. MRPII implementation: Key factors for success. Computer Integrated Manufacturing Systems. N. ${ }^{\circ}$ 5, págs. 31-38. 
ROBEY, D.; ROSS, J.y BOUDREAU, M. 2002.

Learning to implement enterprise systems: An exploratory study of the dialectics of change. Journal of Management Information Systems. Vol. 19, n. ${ }^{\circ}$ 1, págs. 17-46.

ROWE, F. 1999. Cohérence, intégration informationnelle et changement: esquisse d'un programme de recherche á partir des progiciels intégrés de gestion. Systémes d'information et management. Vol. 4, n. ${ }^{\circ}$, pág. 20.

SHANG, S. y SEDDON, P. 2000. A comprehensive framework for classifying the benefits of ERP systems. Long Beach, CA: Proceedings of the 2000 Americas Conference on Information Systems.

SHANKS, G.; PARR, A.; HU, B.; CORBITT, B.; THANASANKIT, T. y SEDDON, P. 2000. Differences in critical success factors in ERP systems implementation in Australia and China: A cultural analysis. Vienna: Proceedings of the $8 .{ }^{\text {th }}$ European Conference on Information Systems.

SHEHAB, E.; SHARP, M.; SUPRAMANIAM, L. y SPEDDING, T. 2004. Entreprise resource planning: An integrative review. Business Process Managerial Journal. Vol. 10, n. ${ }^{\circ}$, págs. 359-386.

STEFANOU, C. 1999. Supply chain management (SCM) and organizational key factors for successful implementation of enterprise resource planning (ERP) systems. Milwaukee, WIS: Proceedings of the Americas Conference on Information Systems.
STRATMAN, J. y ROTH, A. 2002. Enterprise resource planning (ERP) competence constructs: Two-stage multi-item scale development and validation. Decision Sciences. Vol. 33, n. ${ }^{\circ} 4$, págs. 601-628.

SUMNER, M. 1999. Critical success factors in enterprise wide information management systems projects. Milwaukee, WIS: Proceedings of the Americas Conference on Information Systems.

THONG, J. 1999. An integrated model for information systems adoption in SMEs. Journal of Management Information Systems. N. ${ }^{\circ} 15$, págs. 187-214.

VAN EVERDINGEN, Y.; VAN HILLEGERSBERG, J. y WAARTS, E. 2000. ERP adoption by european midsize companies. Communications of the ACM. Vol. 43, n. ${ }^{\circ}$, págs. 27-31.

YEN, D.; CHOU, D. y CHANG, J. 2002. A synergic analysis for web-based enterprise resources-planning systems. Computer Standards \& Interfaces. Vol. 24, n. ${ }^{\circ}$ 4, págs. 337-346.

ZEVALLOS, E. 2003. Micro, pequeñas y medianas empresas en América Latina. Revista de la Cepal. N. ${ }^{\circ} 79$, págs. 53-70.

ZVIRAN, M.; PLISKIN, N. y LEVIN, R. 2005. Measuring user satisfaction and perceived usefulness in the ERP context. Journal of Computer Information Systems. Spring, págs. 43-52. 


\section{Anexo 1 \\ Validez discriminante y confiabilidad de variables-constructos}

\section{Validez discriminante}

Tres de las variables utilizadas fueron medidas mediante constructos del tipo multiítem. En este sentido, el análisis discriminante fue empleado para corroborar la unidimensionalidad de cada uno de ellos. La validez discriminante se verificó empleando la técnica del análisis factorial. Para este caso se utilizó el método de extracción de análisis de componentes principales, mediante rotación Varimax y normalización Kaiser.

A continuación se presenta el reporte estadístico detallado obtenido mediante el uso del paquete estadístico SAS (Enterprise Guide). El cuadro siguiente confirma un valor para el test de Kaiser de 0,71. De acuerdo con Field (2005), un valor mayor que 0,5 ratifica que el análisis factorial es apropiado para la muestra de datos utilizada.

También se verifica que son tres los factores resultantes del análisis, siguiendo la convención referida por Jolliffe (Field, 2005) de mantener aquellos factores cuyos eigenvalores (o valores propios) sean mayores que 0,7 .

Finalmente, se corrobora que todas las variables se adhieren específicamente a uno de los tres factores de acuerdo con la propuesta inicial planteada. Solo surge inconsistencia frente a la clasificación inicialmente propuesta en capacitación en administración del proyecto y rápida instalación del software. Field (2005) argumenta que estas variaciones pueden darse en una muestra limitada y, por tanto, recomienda aumentar el tamaño de la muestra para tener mayor certeza al respecto. 


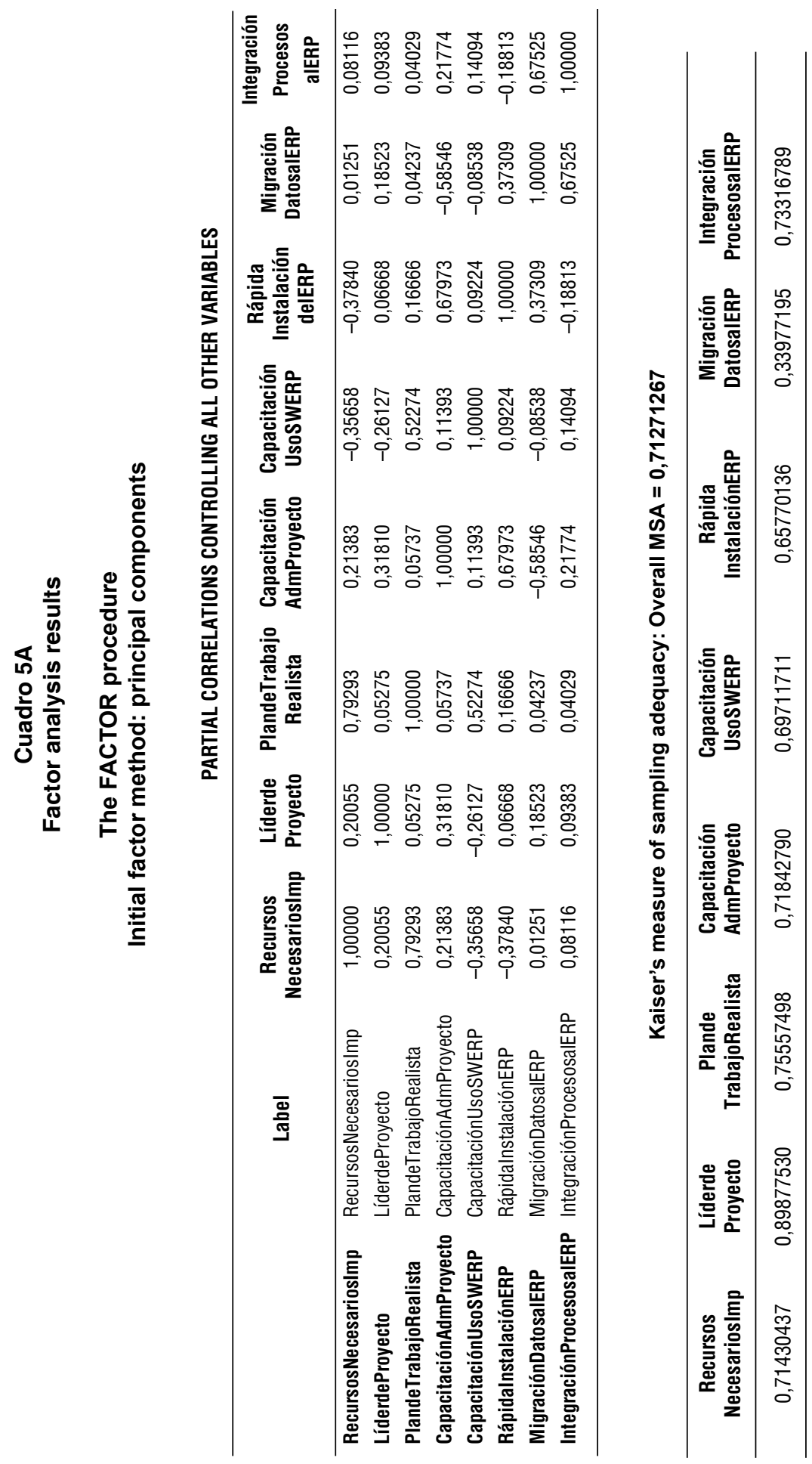



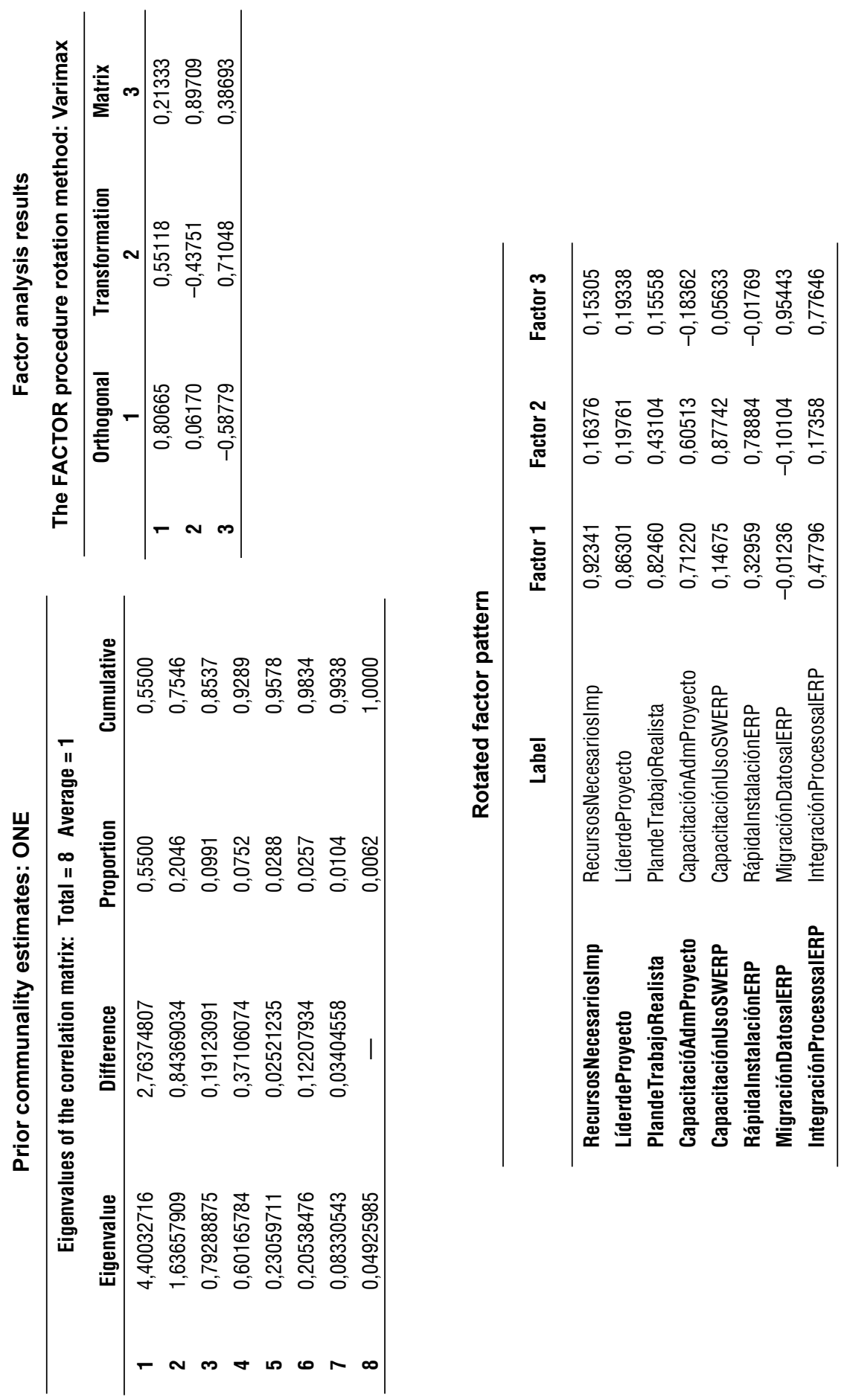


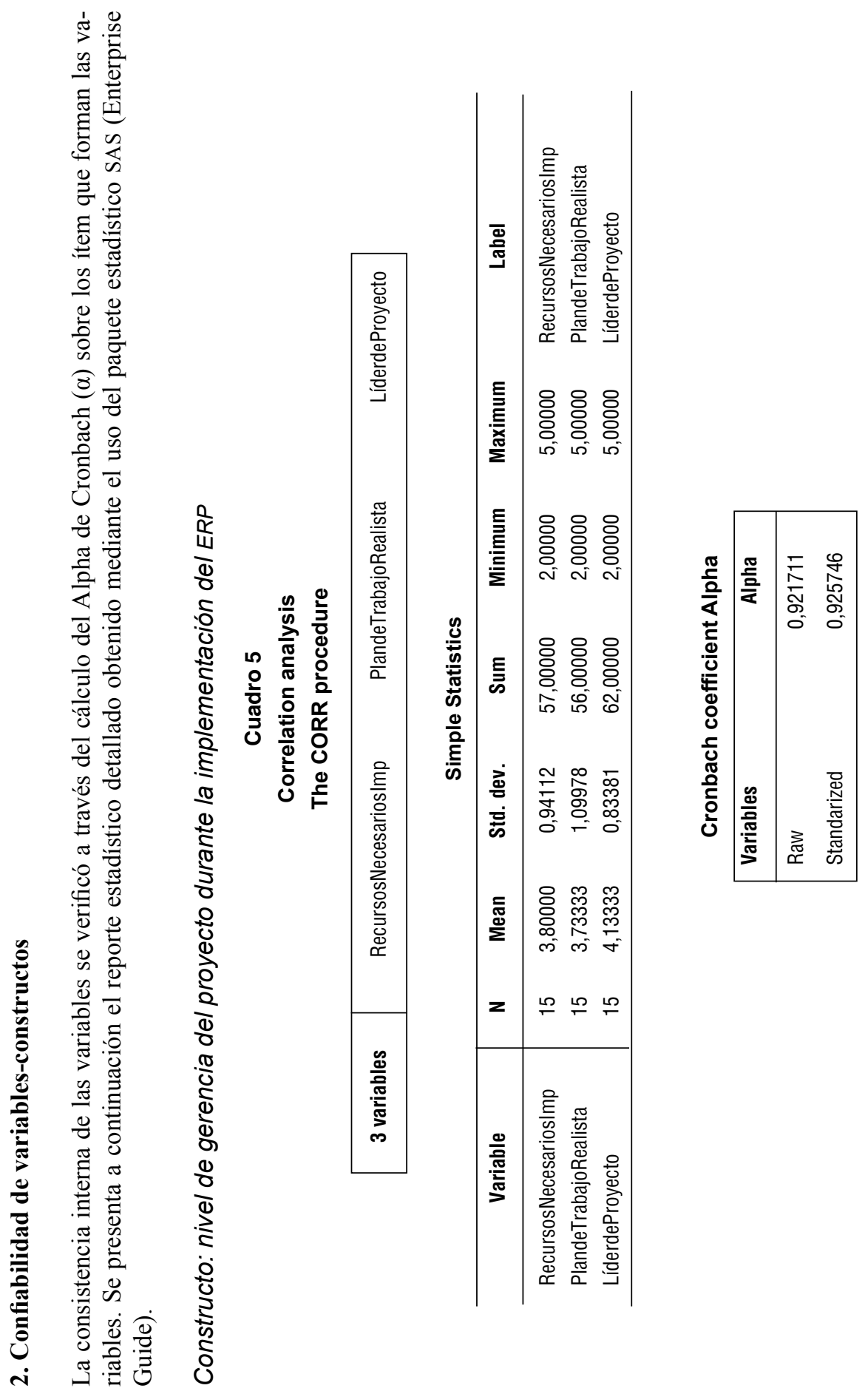




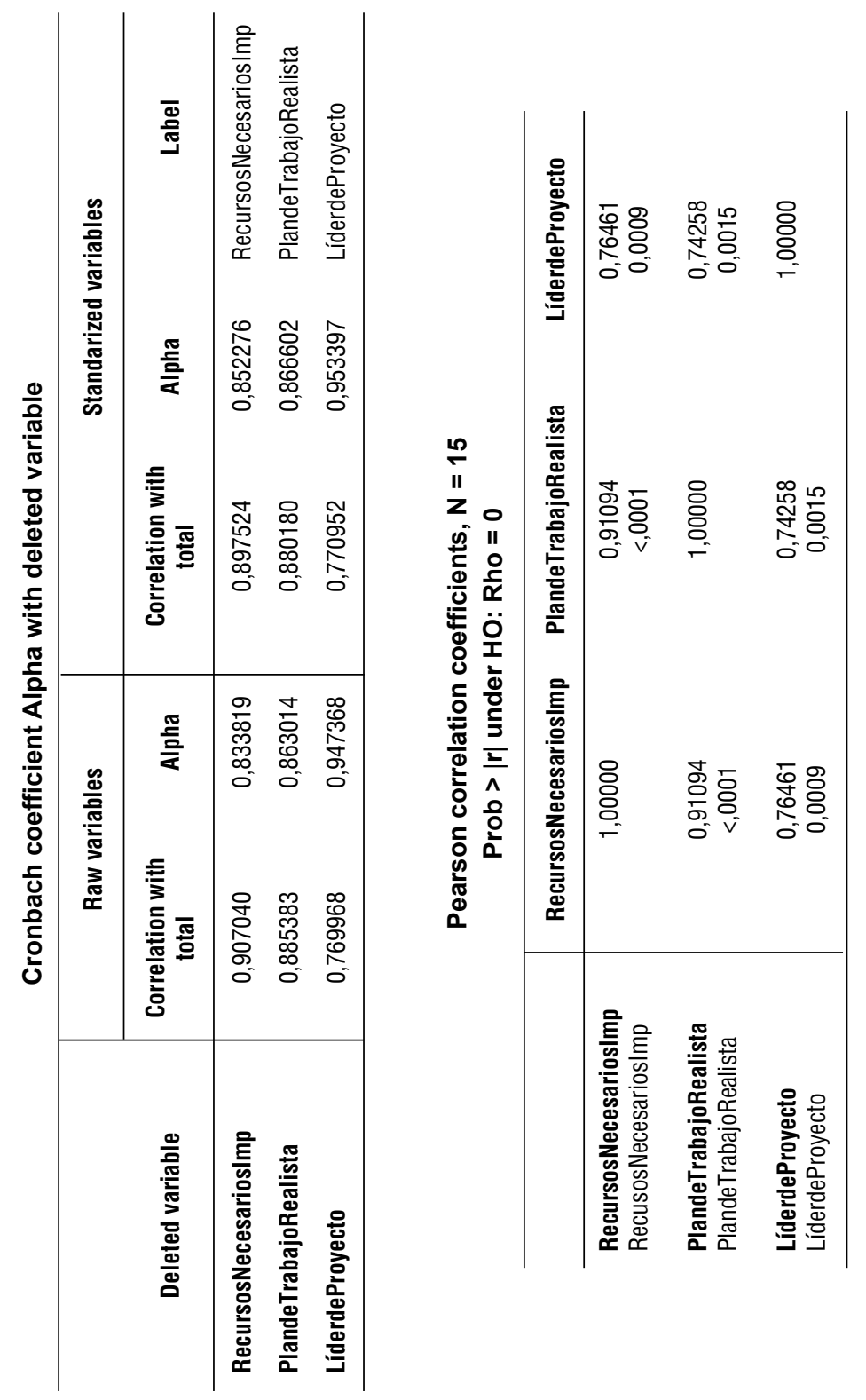




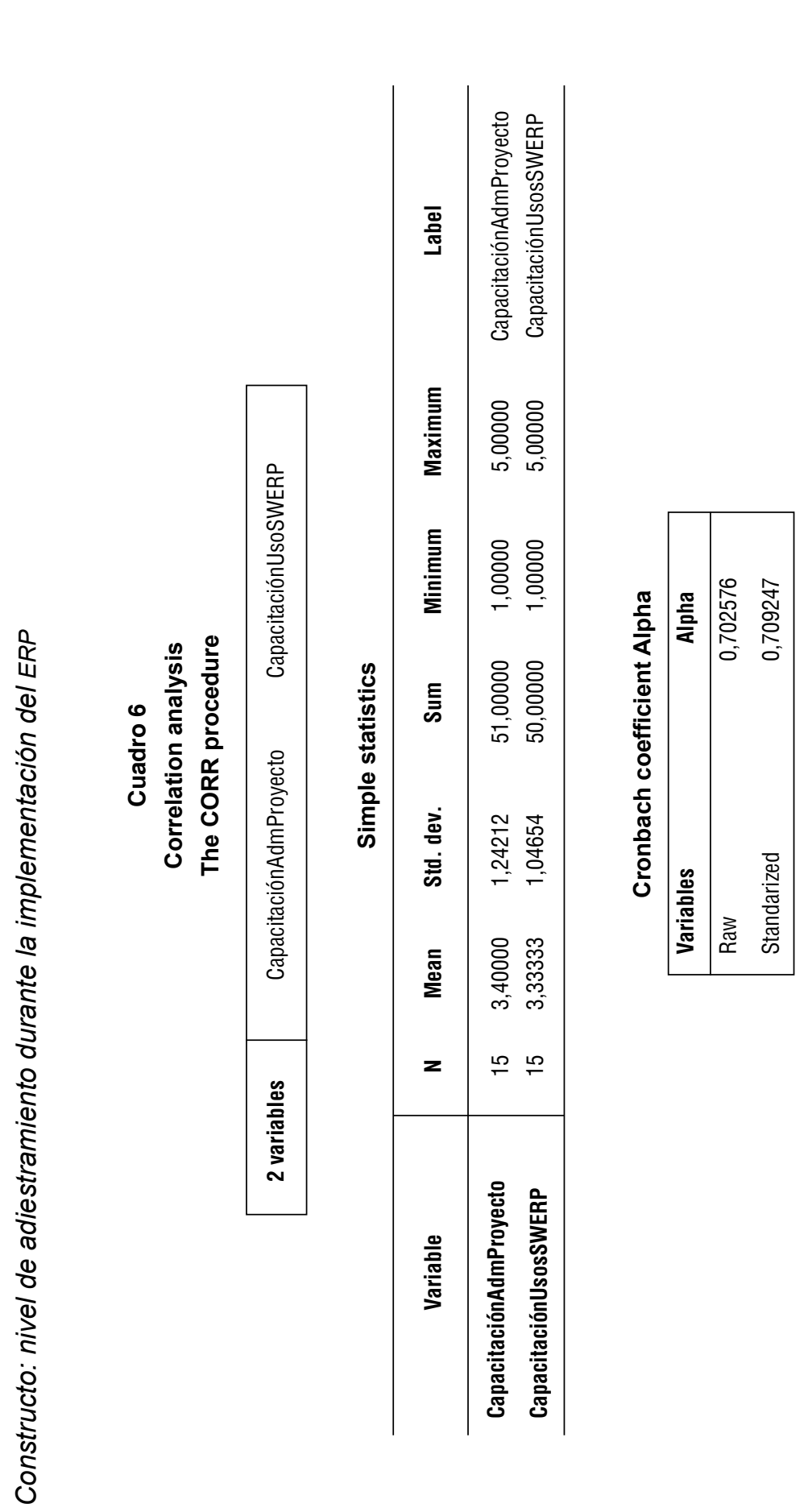




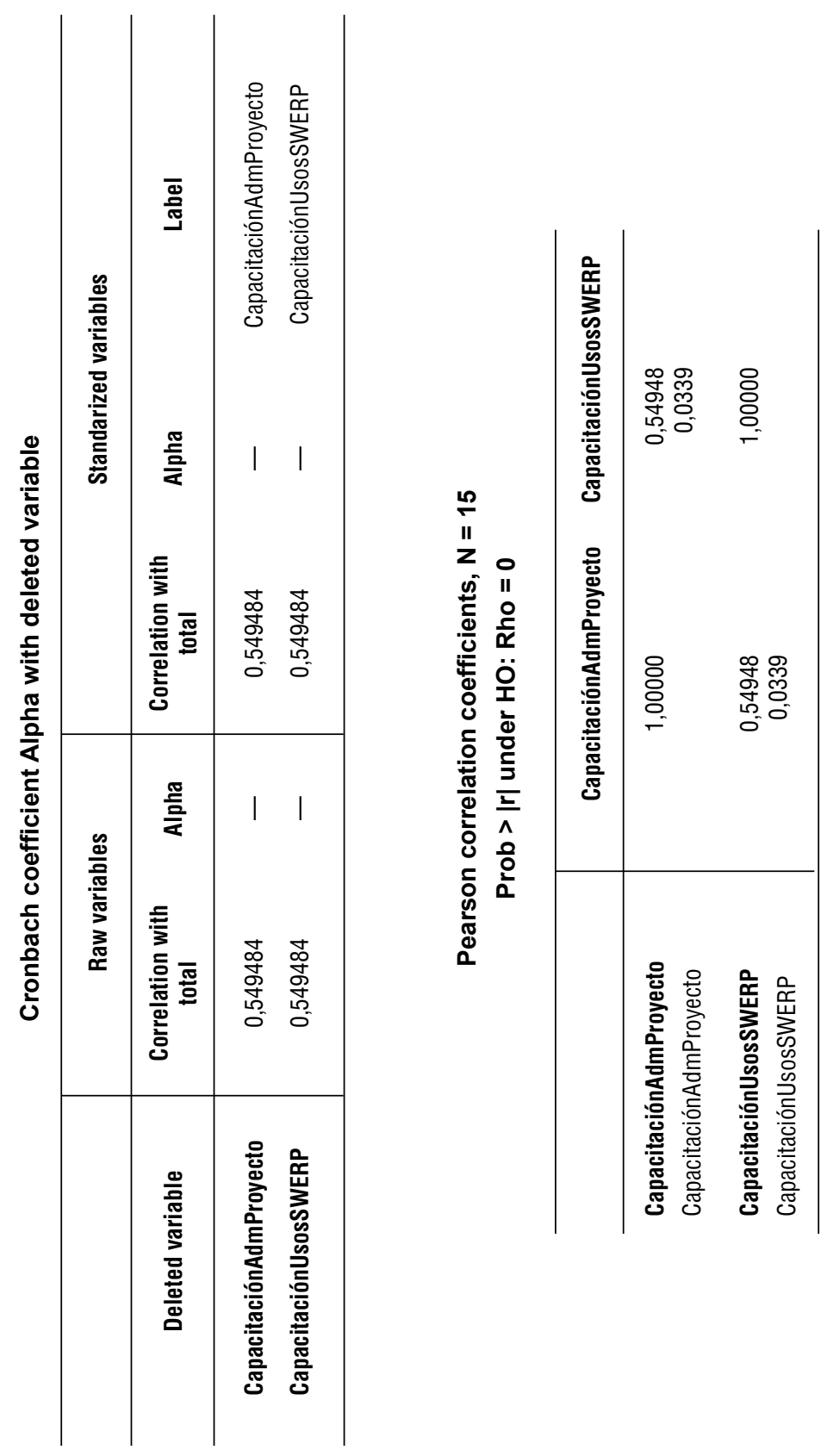




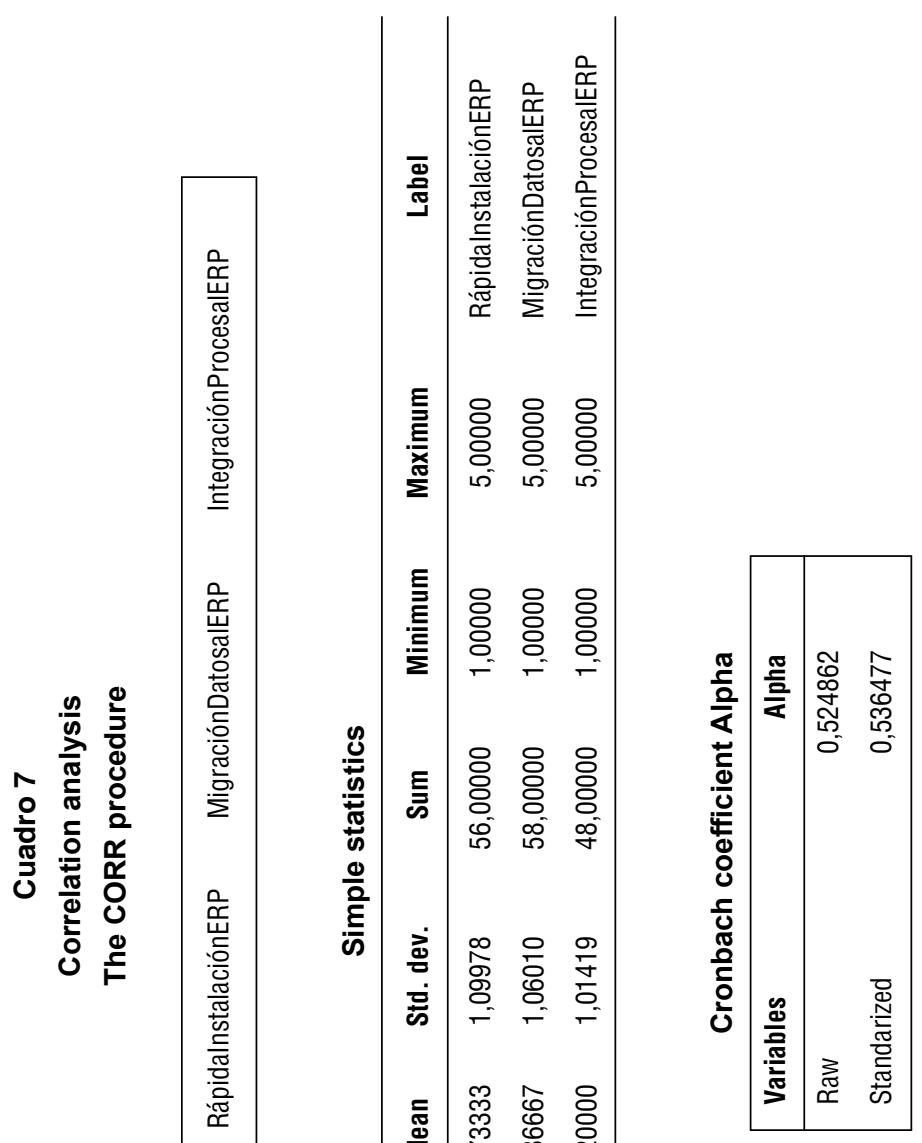

$\frac{1}{6}$

$\frac{\pi}{2}$

응

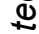

1)

()

$\frac{8}{8}$

$\frac{\sqrt{2}}{3}$

5

ำ 


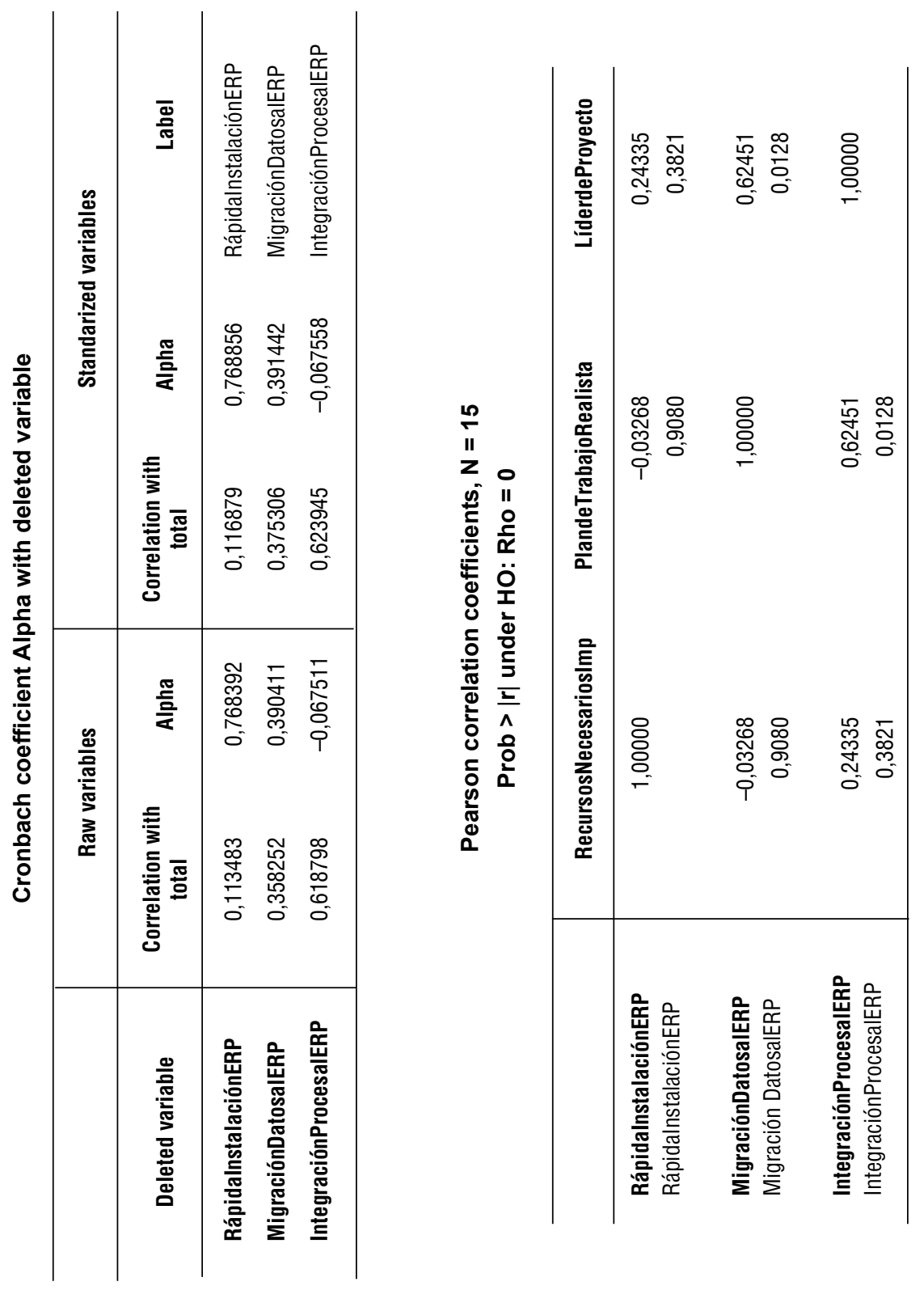




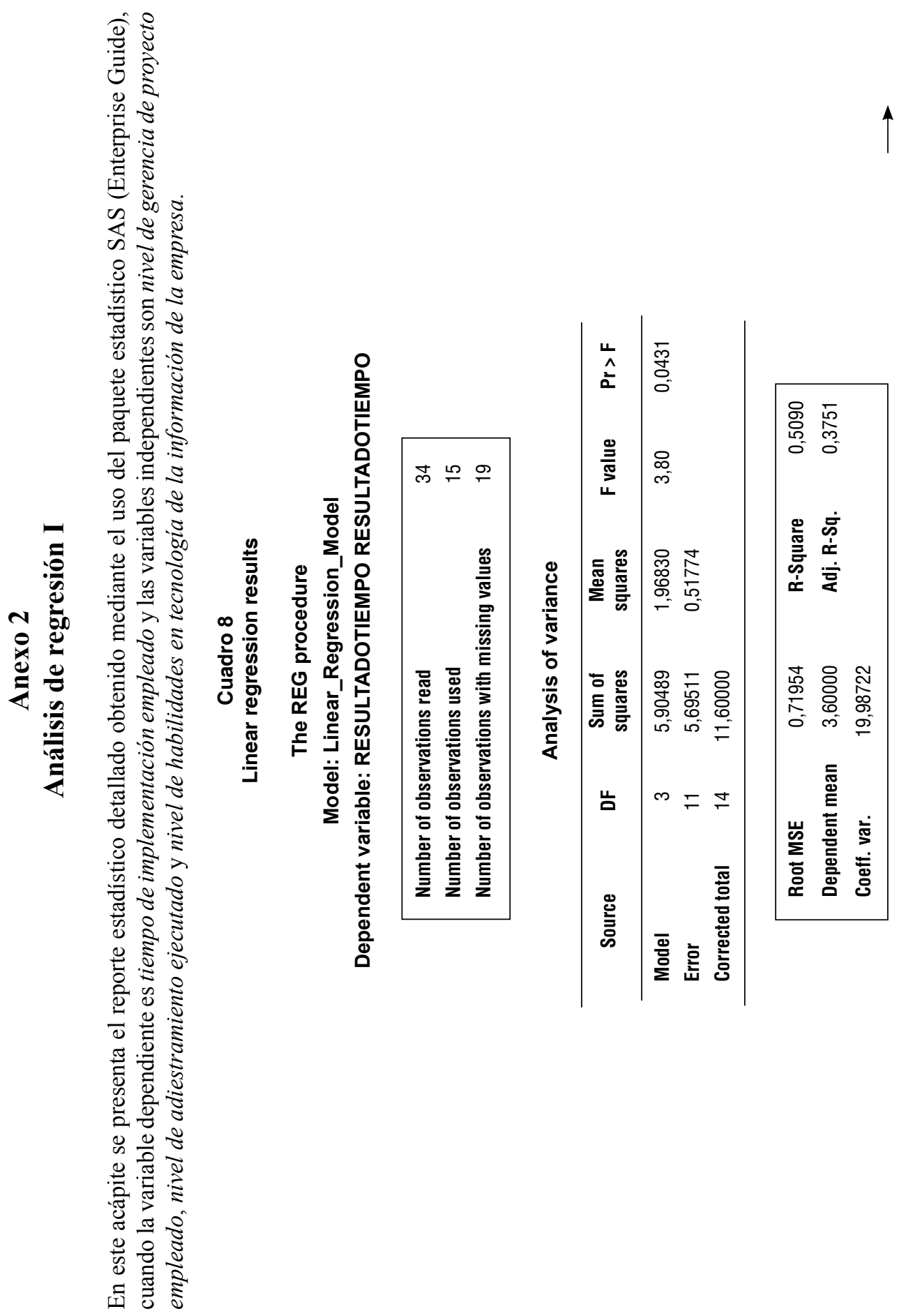



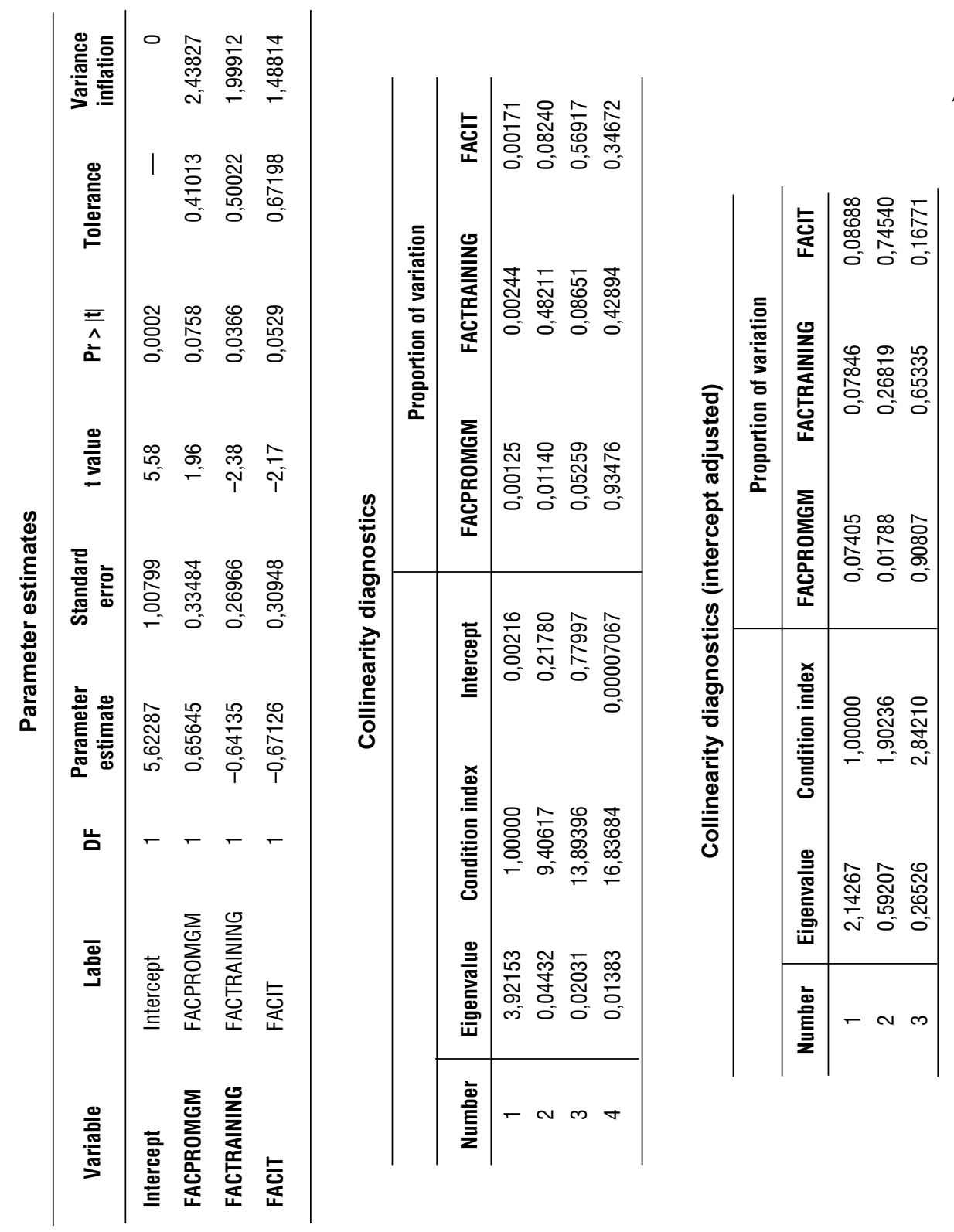


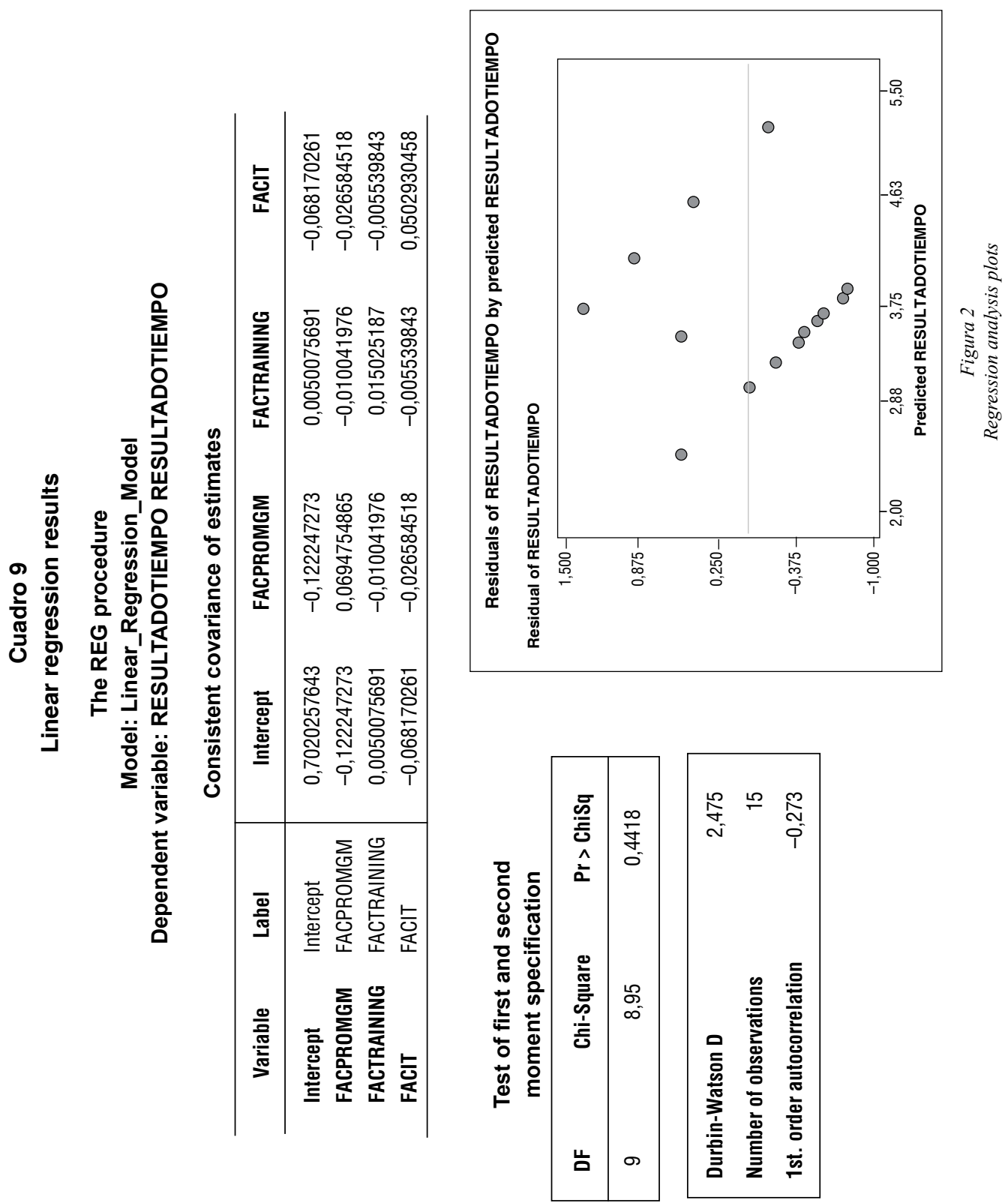




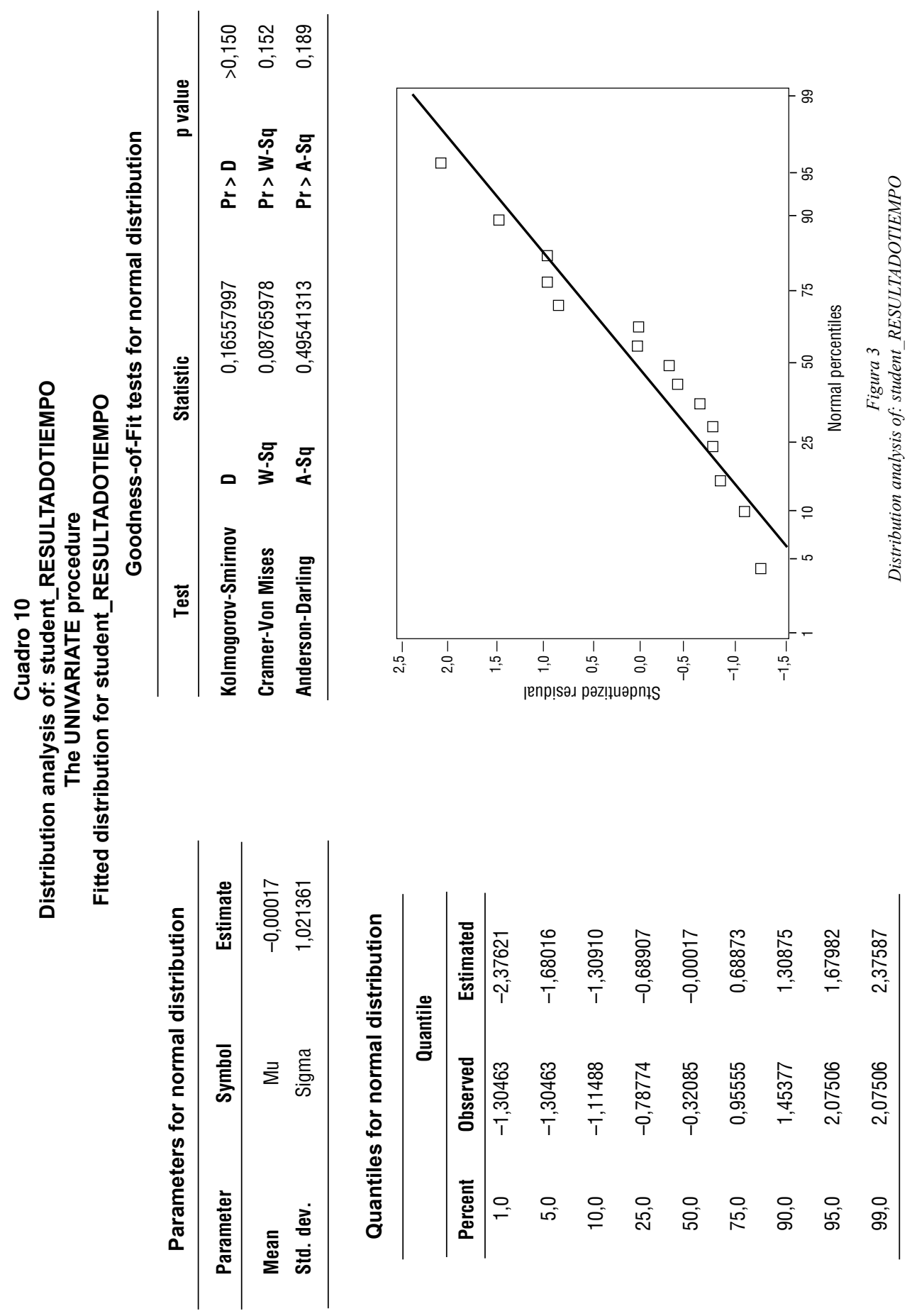




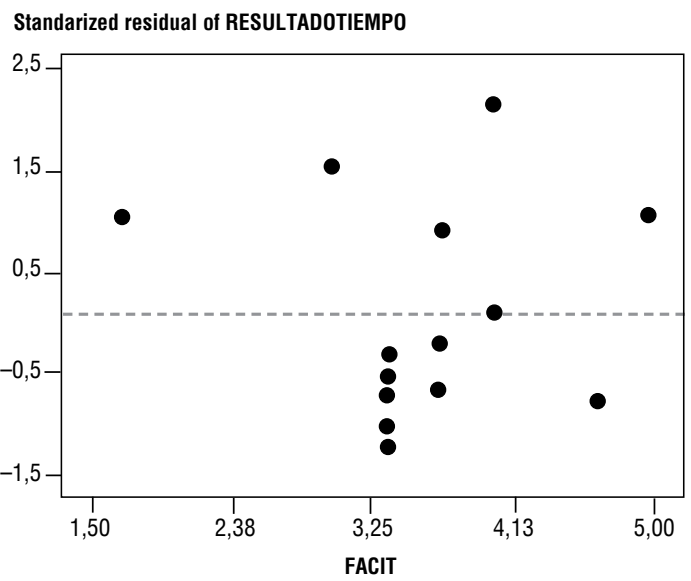

Standarized residual of RESULTADOTIEMPO

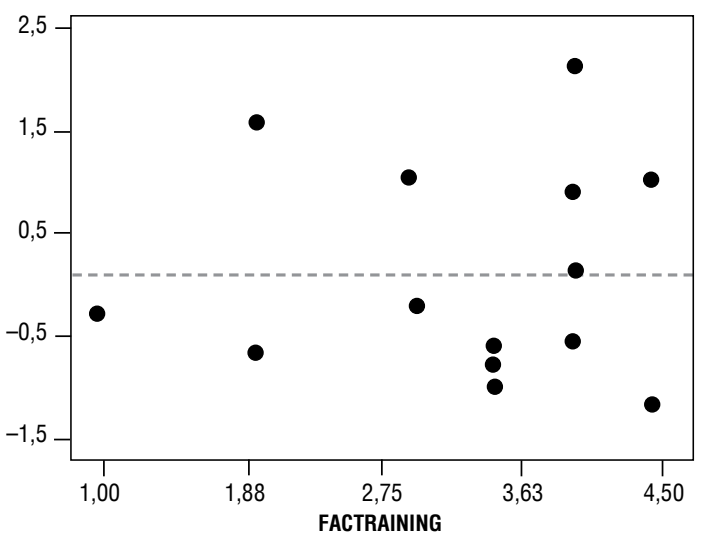

Standarized residual of RESULTADOTIEMPO

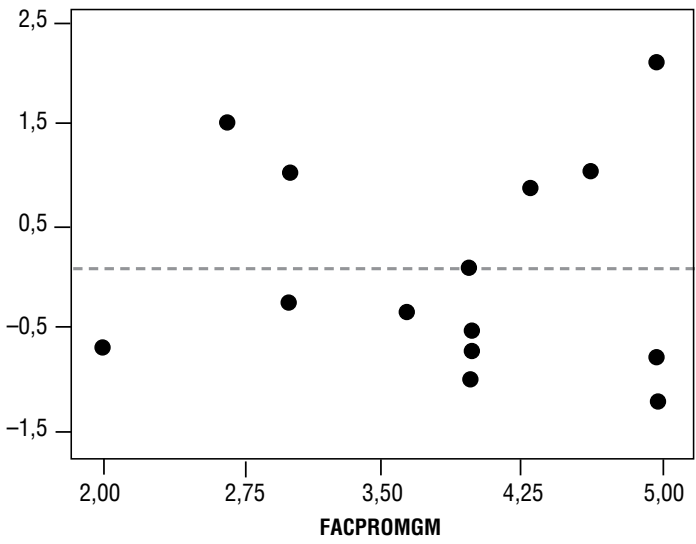

Figura 4

Standarized residual of RESULTADOTIEMPO 


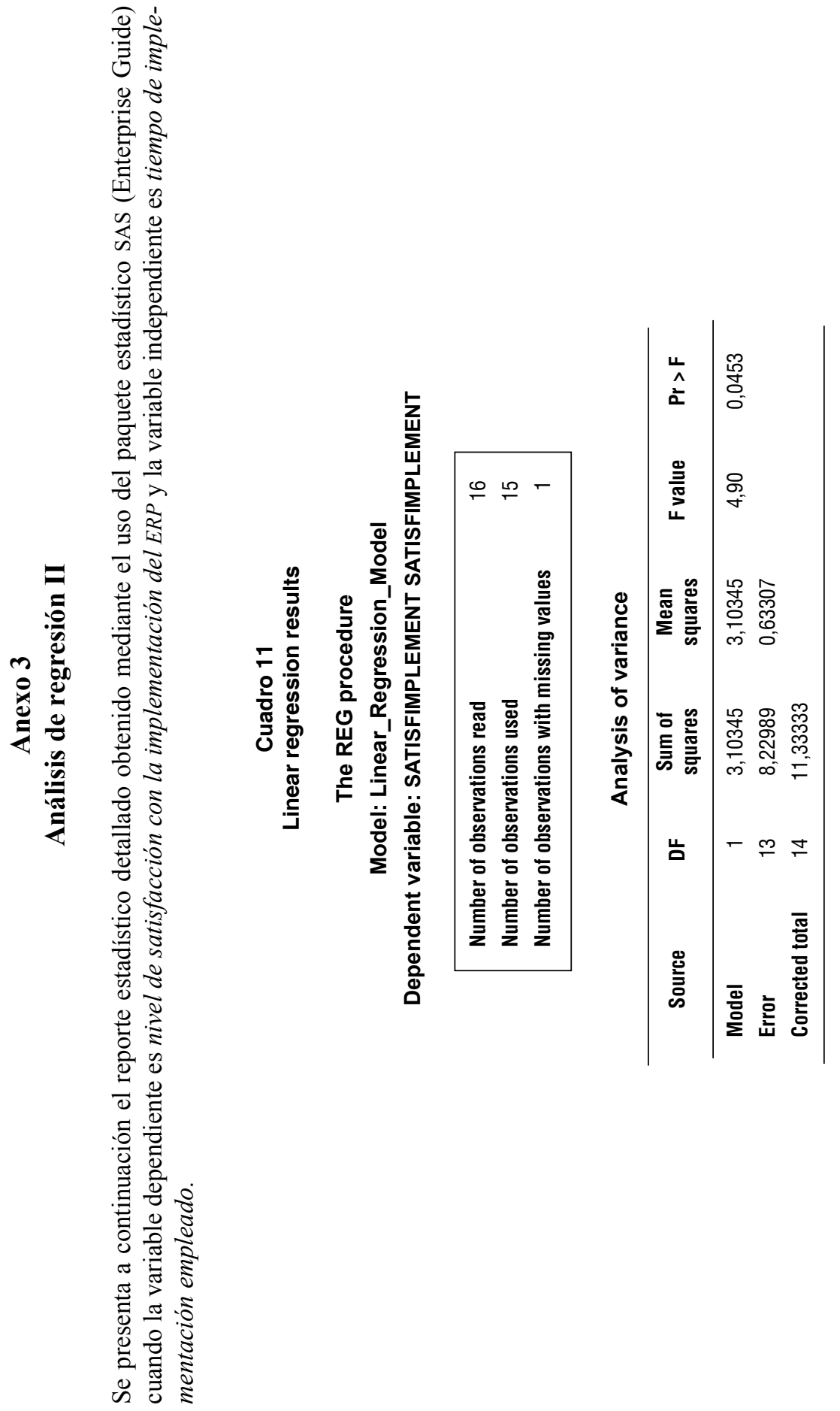




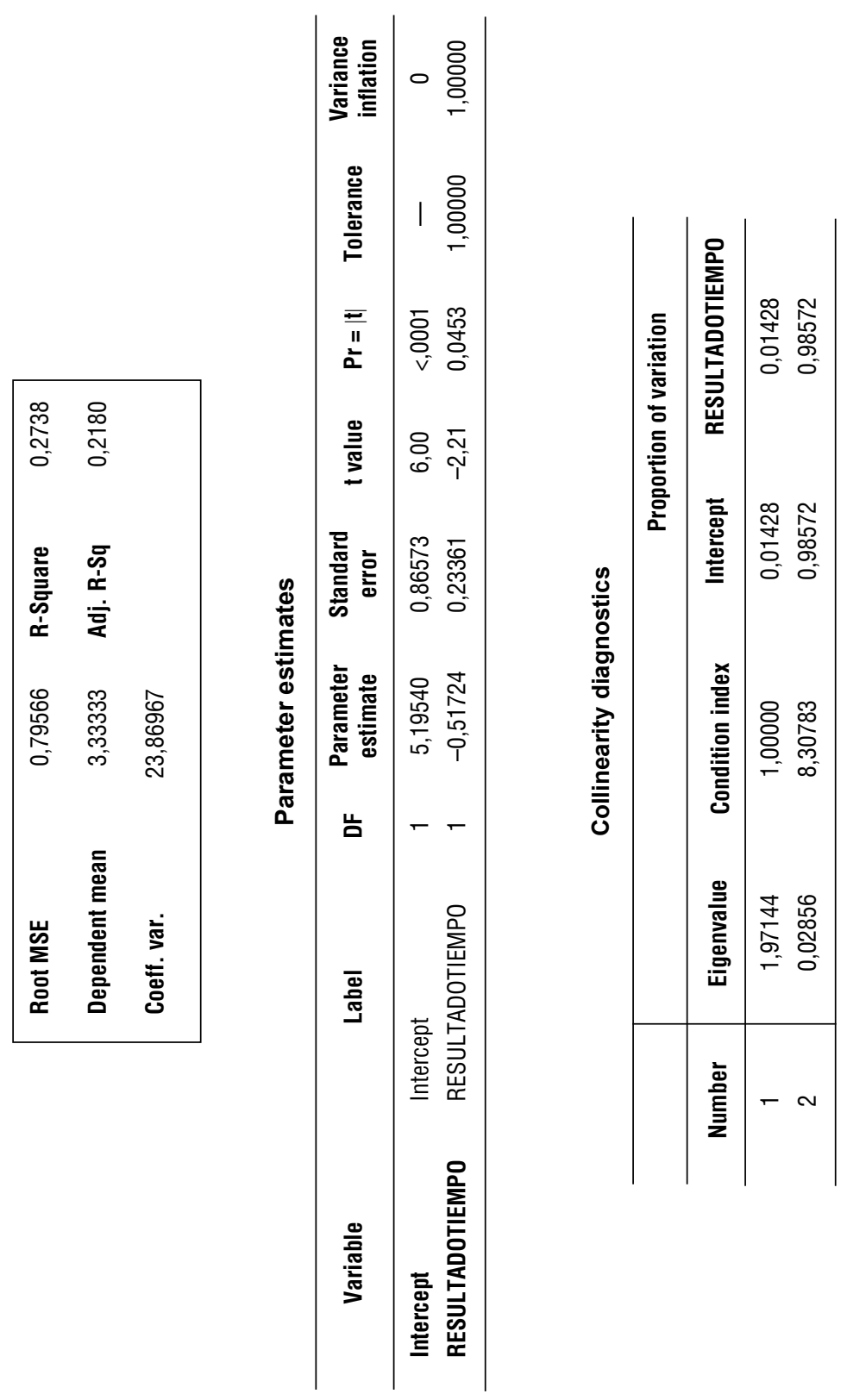




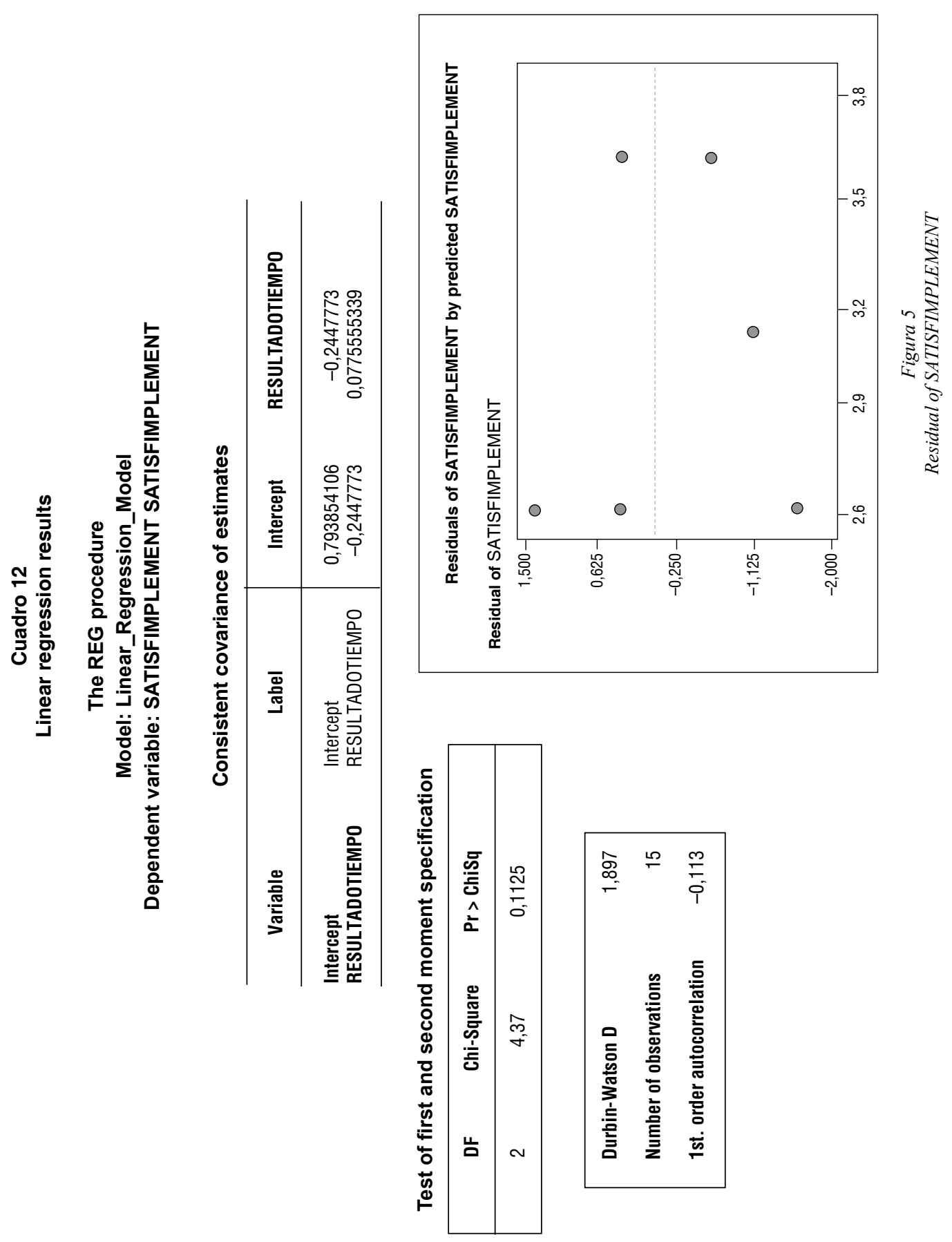




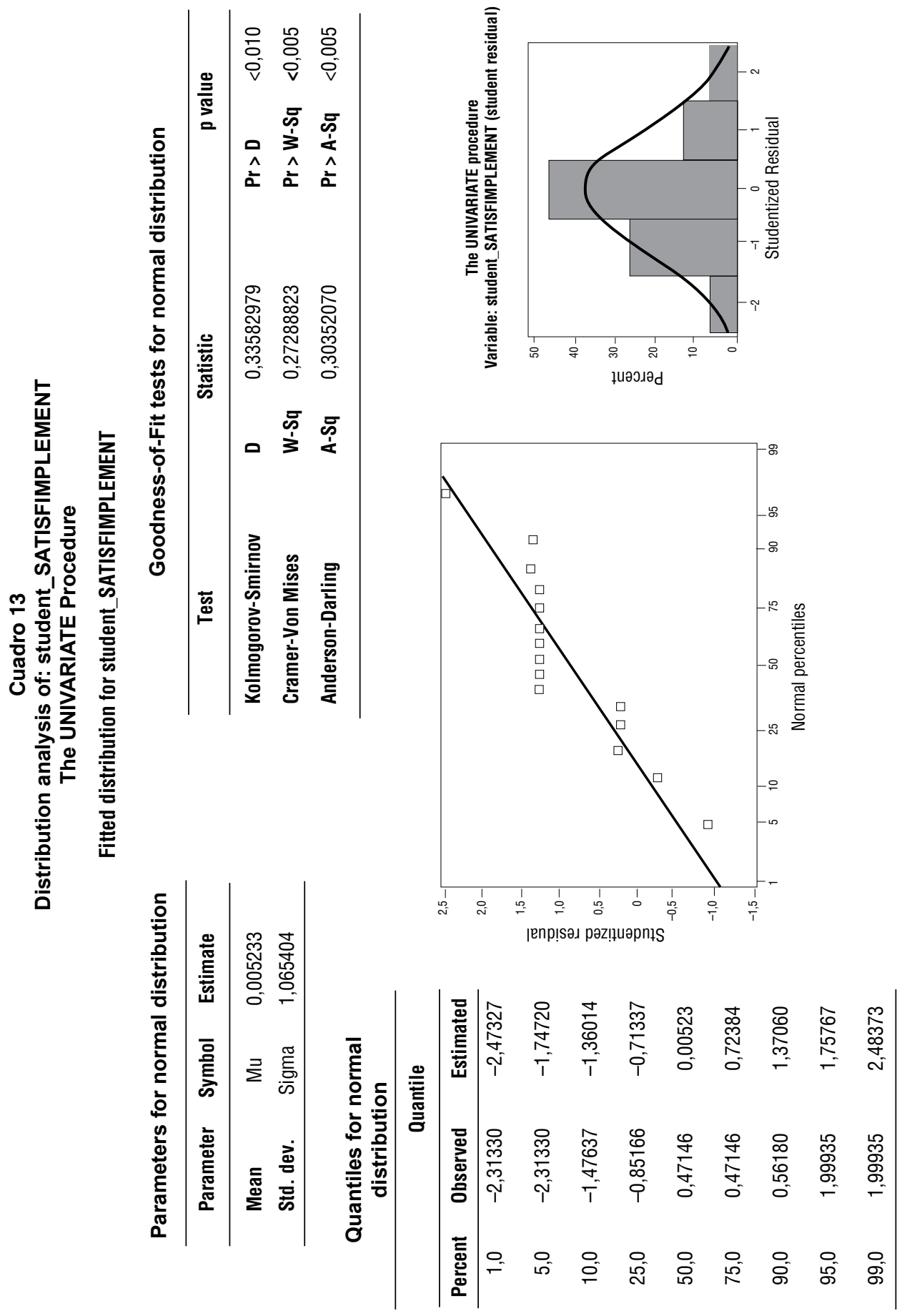




\section{Anexo 4 \\ Cuestionario utilizado}

ESTE ES EL CUESTIONARIO UTILIZADO PARA RECOPILAR LOS DATOS DE LAS EMPRESAS.

Todas las respuestas serán tratadas con absoluta confidencialidad. Es de suma importancia para este estudio que las respuestas a las preguntas reflejen la situación REAL en la empresa y NO lo que hubiese deseado.

Si usted tiene cualquier pregunta con relación a este estudio y la encuesta por favor comuníquese con Miguel Maldonado al correo electrónico:<miguel.maldonadob@gmail.com>.

Una vez contestado el cuestionario favor enviarlo al fax: +1 4198448957 o al e-mail: <miguel. maldonadob@gmail.com>.

¡Muchas gracias por su colaboración!

Con el fin de hacerle llegar una copia del informe final con los resultados del estudio, le rogamos nos proporcione los datos siguientes:

Empresa:

Cargo:

País:

SECCIÓN A. INFORMACIÓN DE LA EMPRESA

Las siguientes preguntas pretenden obtener información general de su empresa. Por favor complete en los espacios con la respuesta que considere más apropiada para cada pregunta.

\section{Por favor indique la naturaleza de su empresa}

a. ¿A qué se dedica su empresa? Manufactura Servicios Comercio Giro / Actividad:

b. ¿Cuántas sucursales tiene la empresa?

N. ${ }^{\circ}$ :

c. ¿Cuál es el tamaño de su empresa? Número de empleados: Facturación: \$

d. ¿Cuándo se fundó la empresa? Año:

e. ¿Cuántas razones sociales (registro fiscal) tiene la empresa? N.o: 
SECCIÓN B. PROCESO DE PREIMPLEMENTACIÓN

Las siguientes preguntas pretenden obtener información sobre las razones que motivaron a su empresa a adquirir un ERP.

De los siguientes motivos para la implantación del ERP ¿cuáles considera usted fueron muy importantes y cuáles no fueron importantes según el entorno de su empresa? (Por favor encierre en un círculo el valor que represente su respuesta según los valores propuestos).

\begin{tabular}{|c|c|c|c|c|c|}
\hline & $\begin{array}{c}\text { Sin } \\
\text { importancia }\end{array}$ & \multicolumn{3}{|c|}{ Neutro } & $\begin{array}{l}\text { Muy } \\
\text { importante }\end{array}$ \\
\hline Presión de los clientes & 1 & 2 & 3 & 4 & 5 \\
\hline $\begin{array}{l}\text { Reemplazar los sistemas de información } \\
\text { anteriores }\end{array}$ & 1 & 2 & 3 & 4 & 5 \\
\hline Mantenerse a la vanguardia tecnológica & 1 & 2 & 3 & 4 & 5 \\
\hline Simplificar y estandarizar los sistemas & 1 & 2 & 3 & 4 & 5 \\
\hline $\begin{array}{l}\text { Mejorar la comunicación y la interacción } \\
\text { con proveedores (presión de proveedores) }\end{array}$ & 1 & 2 & 3 & 4 & 5 \\
\hline $\begin{array}{l}\text { Mejorar la comunicación y la interacción } \\
\text { con clientes (presión de clientes) }\end{array}$ & 1 & 2 & 3 & 4 & 5 \\
\hline $\begin{array}{l}\text { Apoyar una nueva estrategia empresarial } \\
\text { (crecimiento, nuevas líneas de negocio) }\end{array}$ & 1 & 2 & 3 & 4 & 5 \\
\hline $\begin{array}{l}\text { Ligarse y vincularse a actividades/negocios } \\
\text { globales }\end{array}$ & 1 & 2 & 3 & 4 & 5 \\
\hline $\begin{array}{l}\text { Presión de otros competidores en la industria } \\
\text { y el mercado }\end{array}$ & 1 & 2 & 3 & 4 & 5 \\
\hline $\begin{array}{l}\text { Facilidad para actualizar los sistemas } \\
\text { de información en la empresa }\end{array}$ & 1 & 2 & 3 & 4 & 5 \\
\hline Reestructurar la organización de la empresa & 1 & 2 & 3 & 4 & 5 \\
\hline Falta de control & 1 & 2 & 3 & 4 & 5 \\
\hline Necesidad de mejor información & 1 & 2 & 3 & 4 & 5 \\
\hline Otro & 1 & 2 & 3 & 4 & 5 \\
\hline Otro & 1 & 2 & 3 & 4 & 5 \\
\hline
\end{tabular}




\section{SECCIÓN C. PROCESO DE IMPLEMENTACIÓN}

La presente sección pretende investigar algunos factores relevantes en el proceso de implementación del ERP.

A continuación se le presentan algunas acciones y actividades conducidas comúnmente en los proyectos de implementación de ERP en las empresas. Queremos conocer el nivel de satisfacción que usted experimentó con dichas actividades durante la implementación del ERP en su empresa. (Por favor encierre en un círculo el valor que represente su respuesta según los valores propuestos).

\begin{tabular}{|c|c|c|c|c|c|}
\hline & $\begin{array}{c}\text { Nada } \\
\text { satisfecho }\end{array}$ & & Neutro & & $\begin{array}{c}\text { Muy } \\
\text { satisfecho }\end{array}$ \\
\hline $\begin{array}{l}\text { Establecimiento del líder de proyecto de } \\
\text { implementación }\end{array}$ & 1 & 2 & 3 & 4 & 5 \\
\hline $\begin{array}{l}\text { Adecuada y suficiente capacitación del } \\
\text { software ERP }\end{array}$ & 1 & 2 & 3 & 4 & 5 \\
\hline $\begin{array}{l}\text { Rápida y eficiente instalación del software } \\
\text { ERP en la empresa }\end{array}$ & 1 & 2 & 3 & 4 & 5 \\
\hline Definición de un Plan de Trabajo realista & 1 & 2 & 3 & 4 & 5 \\
\hline $\begin{array}{l}\text { Migración de los datos existentes en la } \\
\text { empresa a la nueva plataforma ERP }\end{array}$ & 1 & 2 & 3 & 4 & 5 \\
\hline $\begin{array}{l}\text { Integración eficiente de los procesos de } \\
\text { negocio al software ERP }\end{array}$ & 1 & 2 & 3 & 4 & 5 \\
\hline $\begin{array}{l}\text { Adecuada y suficiente capacitación sobre } \\
\text { gerencia de proyectos ERP }\end{array}$ & 1 & 2 & 3 & 4 & 5 \\
\hline $\begin{array}{l}\text { Claro establecimiento de los recursos } \\
\text { necesarios durante la implementación }\end{array}$ & 1 & 2 & 3 & 4 & 5 \\
\hline
\end{tabular}




\section{SECCIÓN D. SATISFACCIÓN CON EL PROCESO DE IMPLEMENTACIÓN DEL ERP}

Las siguientes preguntas pretenden conocer su satisfacción con la implementación del ERP en su empresa.

Por favor encierre en un círculo el valor que represente su respuesta según los valores propuestos.

Con respecto al tiempo real que se tomó la implementación del ERP en su empresa y las expectativas inicialmente planteadas, por favor indique el valor que más se aproxime a su criterio encerrando en un círculo el valor elegido.

\begin{tabular}{|c|c|c|}
\hline $\begin{array}{c}\text { El tiempo de } \\
\text { implementación resultó } \\
\text { muy por debajo de lo } \\
\text { esperado }\end{array}$ & $\begin{array}{c}\text { El tiempo de } \\
\text { implementación se } \\
\text { ajustó a lo esperado }\end{array}$ & $\begin{array}{c}\text { El tiempo de } \\
\text { implementación fue } \\
\text { muy excedido a lo } \\
\text { esperado }\end{array}$ \\
\hline 1 & 2 & 5 \\
\hline
\end{tabular}

Con respecto al nivel de satisfacción general de su empresa con el proceso de implementación del ERP ¿cómo lo calificaría de acuerdo a la escala que se le presenta? Favor indique el valor que más se aproxime a su criterio encerrando en un círculo el valor elegido.

$\begin{array}{ccc}\text { Nada satisfecho } & \text { Ni satisfecho ni } & \text { Muy satisfecho } \\ \text { con el proceso de } & \text { insatisfecho con } & \text { con el proceso de } \\ \text { implementación del } & \text { el proceso de } & \text { implementación del } \\ \text { ERP en la empresa } & \text { implementación del } & \text { ERP en la empresa } \\ & \text { ERP en la empresa } & \end{array}$

\begin{tabular}{llllll}
\hline 1 & 2 & 3 & 4 & 5 \\
\hline
\end{tabular}

\section{ESPACIO PARA COMENTARIOS ADICIONALES}

Por favor, formule aquí cualesquiera comentarios juzgue pertinentes, ya sea sobre aspectos no cubiertos en este cuestionario, como sobre este cuestionario mismo y/o sobre aspectos de importancia para su sector/segmento.

Muchas gracias por completar este cuestionario. Toda la información proporcionada será manejada de forma estrictamente confidencial. 